\title{
Molecule guided laser ablation as a novel therapeutic strategy to control itch.
}

Linda Nocchi ${ }^{1}{ }^{2}$, Mariangela D'Attilia ${ }^{1}$, Nainika Roy ${ }^{1}$, Rahul Dhandapani ${ }^{1}{ }^{12}$, Andrei Traista ${ }^{1}$, Mariano Maffei ${ }^{1,2}$, Laura Castaldi ${ }^{1,2}$, Emerald Perlas ${ }^{1}$, Paul A. Heppenstall1,2,*

${ }^{1}$ EMBL Rome, Via Ramarini 32, Monterotondo 00015, Italy.

${ }^{2}$ Molecular Medicine Partnership Unit (MMPU), Heidelberg, Germany.

${ }^{*}$ Correspondence to:

Paul Heppenstall, EMBL Rome, Via Ramarini 32, Monterotondo 00015, Italy. Tel: +39 0690091233, Fax: +390690091272, Email: paul.heppenstall@embl.it 


\section{Abstract}

Itch is a major symptom of many chronic skin diseases that can exacerbate inflammation by provoking excessive scratching and causing skin damage. Here we develop a novel technology to control itch through molecular guided delivery of a phototoxic agent and near infrared (IR) illumination of the skin. Exploiting the selective binding of the pruritogen Interleukin-31 to itch sensing cells, we generate an engineered IL31 SNAP ligand derivative (IL31 ${ }^{\mathrm{K} 138 \mathrm{~A}-\mathrm{SNAP}}$ ) that binds to cells but does not evoke signaling or provoke scratching when injected in vivo. Conjugation of IL31 K138A-SNAP to the photosensitizer IRDyeß700DX phthalocyanine (IR700) and injection of the skin results in long-term reversal of scratching behavior evoked by IL31 upon near IR illumination. We further develop a topical preparation of IL31 K138A-SNAP_IR700 that strikingly, reverses behavioral and dermatological indicators in mouse models of Atopic Dermatitis (AD) and the genetic skin disease Familial Primary Localized Cutaneous Amyloidosis (FPLCA). We demonstrate that this therapeutic effect results from selective retraction of itch sensing neurons in the skin, breaking the cycle of itch and disruption of the skin's barrier function. Thus, molecule guided photoablation is a powerful new technology for controlling itch and treating inflammatory skin diseases.

\section{Introduction}

Itch is a cutaneous sensory perception defined by the behavioral response it elicits: an urgent need to scratch ${ }^{1}$. When itching becomes pathological, it can be irritating and distressful and have a dramatic impact on quality of life ${ }^{2}$. Chronic itch generates a recurrent cycle whereby the more the skin is scratched, the more it itches ${ }^{3}$. In turn, this may lead to serious damage to the skin barrier and thereby an increased risk of infection ${ }^{3}$. There are many itch-associated diseases such as Atopic Dermatitis (AD) and psoriasis that respond poorly to current therapies ${ }^{4}$. Identifying novel strategies to reduce itching is therefore critical and requires a deeper understanding of the underlying mechanisms.

Although several key molecules involved in transducing itch sensation have recently been described, the cellular and molecular mechanisms that drive chronic itch are not fully understood. The transduction of itch begins in the skin where a network of different cell types, such as keratinocytes, sensory nerves and immune cells respond to exogenous or endogenous pruritogens and initiate the cascade which ends with the scratch response ${ }^{5}$. Two major itch pathways have been described: the histaminergic pathway which is mediated 
by histamine ${ }^{6}$ and the non-histaminergic pathway which includes a plethora of other itch mediators, such as chloroquine, neuropeptides and inflammatory cytokines ${ }^{7}$. It is becoming increasingly apparent that anti-histaminergic drugs are often ineffective against many chronic itch conditions such as Atopic Dermatitis and psoriasis ${ }^{8,9}$. Attention has now turned to non-histaminergic pathways to control itch sensation under pathological conditions ${ }^{10}$.

Amongst anti-histamine resistant itch mediators, the cytokine Interleukin 31 (IL-31) has recently attracted much attention as a novel target molecule for chronic itch therapy ${ }^{11}$. IL31 is a cytokine with a prominent skin tropism that is produced preferentially by $\mathrm{T}$ helpertype 2 cells ${ }^{12}$. It signals through a heterodimeric receptor composed of IL31RA and OSMR which are expressed in epithelial cells, keratinocytes and sensory neurons ${ }^{13,14}$. Transgenic mice overexpressing IL-31 develop severe pruritus, alopecia and skin lesions that resemble lesioned skin from patients with atopic dermatitis ${ }^{11}$. Moreover, numerous studies have reported an association of IL-31 with inflammatory skin diseases with a severe pruritic component such as atopic dermatitis, prurigo nodularis, allergic contact dermatitis, and cutaneous T-cell lymphoma ${ }^{15-18}$. For example, IL-31 mRNA is up-regulated in human patients and mouse models of atopic dermatitis ${ }^{15,19}$ and increased levels of IL31 correlate with disease severity ${ }^{20,21}$. A common IL31 haplotype has also been associated with nonatopic eczema in three independent European populations, marking this as the first genetic risk factor for non-atopic eczema ${ }^{22}$. Furthermore, mutations in the IL31 receptors IL31RA and OSMR underlie Familial Primary Localized Cutaneous Amyloidosis (FPLCA), a hereditary skin disorder characterized by the deposition of amyloid in the dermal papilla and severe pruritus ${ }^{23-27}$. Intriguingly, it has been suggested that the major pathology evoked by IL-31 is to induce pruritus, rather than directly causing skin lesions per se ${ }^{28}$. Thus, therapies which reduce scratching and break the cycle of itch and disruption of the skin's barrier function ${ }^{29}$ may be the most effective strategies for improving the quality of life for patients with chronic pruritic disease ${ }^{3}$. Indeed, recent phase I and 2 clinical trials of a humanized antibody against IL31RA (Nemolizumab, CIM331) have shown some promise in improving pruritus in patients with moderate to severe atopic dermatitis ${ }^{30,31}$.

Here we have asked whether the IL31 ligand may serve as an alternative means of accessing pathways in the skin which provoke itch. Because IL31 receptors are expressed by itch sensing cells, we reasoned that conjugation of the IL31 ligand to a photosensitizer may allow for specific delivery of phototoxic complexes to these cells in the skin, and thus enable light activated suppression of itch sensation at its source. To achieve this aim we generated a recombinant IL31 fusion protein with a SNAP tag placed at its C-terminus that 
would allow for its chemical modification with benzylguanine (BG) derivatized probes. We demonstrate that IL31 SNAP is functional and can be conjugated to a photosensitizer to control IL31-induced scratching behavior. We further identify an IL31 mutant (IL31 ${ }^{\mathrm{K} 138 \mathrm{~A}}$ ) that binds to keratinocytes but no longer provokes signaling or scratching. Delivery of IL31 K138A-SNAP IR700 to the skin and subsequent near infrared illumination inhibits acute scratching and reverses behavioral and dermatological indicators in mouse models of Atopic Dermatitis and FPLCA. Intriguingly, this effect appears to be dependent upon retraction of sensory fibers from the epidermis of the skin, thus breaking the cycle of itch and disruption of the skin's barrier function.

\section{Results}

Generation and characterization of IL31SNAP

Recombinant IL31 was produced with a C-terminal fusion of SNAP (IL31 SNAP) in E. Coli. Following purification and refolding from inclusion bodies, IL31 SNAP was efficiently labelled with BG derivatized fluorophores (Figure 1a) indicating that the SNAP tag was successfully expressed and correctly folded in the fusion protein. To determine whether IL31 SNAP was functional we first performed binding studies in primary keratinocyte cultures. IL31 SNAP was labelled in vitro with BG-Surface549 and applied to keratinocytes from wildtype or IL31 Receptor A (IL31RA) knockout mice (IL31RA ${ }^{-/}$). At a range of concentrations we observed strong fluorescent signal internalized in wildtype keratinocytes that was not present in cells for IL31RA ${ }^{-/}$mice (Figure $1 \mathrm{~b}$ and $\mathrm{c}$ and Supplementary Figure $1 \mathrm{a}-\mathrm{k}$ ). We further examined whether IL31 SNAP was active by quantifying scratching behavior in mice upon intradermal injection. IL31 ${ }^{\text {SNAP }}$ evoked robust scratching that was comparable in duration and intensity to native recombinant IL31 in wildtype mice (Figure 1d and Supplementary Figure 1I). In IL31RA ${ }^{-/}$mice, IL31 ${ }^{\text {SNAP }}$ and IL31 did not evoke scratching (Figure 1d). Thus, the IL31 SNAP retains the functional properties of native IL31.

\section{IL31 mediated photoablation}

To manipulate IL31 receptor expressing cells in vivo, we reasoned that IL31SNAP may allow for targeted photoablation of these cells through delivery of a photosensitizing agent. We synthesized a benzylguanine modified derivative of the highly potent near-infrared photosensitizer IRDye ${ }^{8} 700 D X$ phthalocyanine (IR700) and conjugated it in vitro to IL31 SNAP 32. Application of IL31 SNAP-IR700 to keratinocytes followed by 1 minute illumination provoked substantial cell death (Figure 1e) that was not evident in keratinocytes only treated with 
IR700 + illumination (Figure 1f) or in keratinocytes from IL31RA ${ }^{-/-}$mice (Figure 1g). We further examined photosensitizer effects in vivo by injecting IL31SNAP-IR700 subcutaneously, applying near infrared light to the skin, and monitoring IL31 evoked scratching behavior. Strikingly, a progressive decrease in scratching bouts was observed when IL31 SNAP_IR700 was injected for three consecutive days and the skin illuminated (Figure 1h).

\section{Generation and characterization of a non-signaling IL31 mutant}

A conceptual problem of using IL31SNAP therapeutically is that it in itself evokes itch. We therefore sought to engineer IL31 SNAP to obtain a ligand that still binds to IL31 receptor complex but no longer promotes signaling. From a previous structural study ${ }^{33}$ we selected an IL31 point mutant IL31 ${ }^{\mathrm{K} 134 \mathrm{~A}}$ that was reported to exhibit reduced signaling in cells expressing human IL31 receptors. We generated a recombinant mouse IL31 K138A-SNAP fusion protein (orthologous to human IL31 ${ }^{\mathrm{K} 134 \mathrm{~A}}$ ), labelled it with BG-Surface549 and applied it to keratinocytes. Pronounced fluorescence was evident in cells from wildtype mice treated with fluorescent IL31 K138A-SNAP (Figure 2a), at a similar concentration range to that observed with IL31 SNAP (Supplementary Figure 2a-k). Importantly, such signal was not present in IL31RA1- keratinocytes (Figure 2b). We further assessed cellular signaling pathways activated by IL31 ${ }^{\text {SNAP }}$ and IL31 K138A-SNAP in the skin by examining levels of phosphorylated Akt, pMAPK and pSTAT3 which have all been previously implicated in IL31 downstream signaling 14, 33, 34. Mice were injected subcutaneously with IL31 SNAP and IL31 K138A-SNAP and skin harvested 1 hour later for immunoblot analysis. We observed an increase in phosphorylation of the downstream pathways in skin injected with IL31 SNAP and this increase was absent in skin injected with IL31 K138A-SNAP (Figure 2c and d). As a final test for the functional activity of IL31 ${ }^{\mathrm{K} 138 \mathrm{~A}-\mathrm{SNAP}}$, we assayed its capacity to provoke scratching behavior in mice. In contrast to IL31 SNAP which induced robust scratching when injected intradermal, IL31 K138A-SNAP did not evoke any scratching above baseline levels in mice (Figure $2 \mathrm{e}$ ). Thus, the engineered ligand IL31 K138A-SNAP may offer a powerful means of targeting cells involved in itch without triggering itch in itself.

\section{IL31K138A-SNAP-IR700 mediated photoablation and acute itch}

To characterize IL31 ${ }^{\mathrm{K} 138 \mathrm{~A}-\mathrm{SNAP}}$ mediated photoablation in vivo, we first tested its efficacy at alleviating IL31 provoked itch. Mice were treated for three consecutive days with IL31K138ASNAP-IR700 and the skin was illuminated with near-IR light. Strikingly, IL31-induced scratching behavior was abolished in these animals (Figure 2f), and this persisted throughout an 8 week observation period (Supplementary Figure 2I). In control mice that

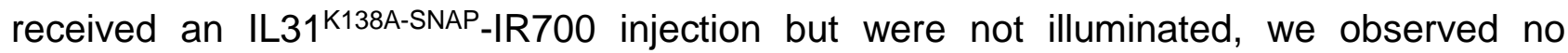


reduction in IL31-evoked scratching (Figure 2f). We next tested the effects of photoablation on scratching provoked by other acutely applied pruritogens. Intriguingly, IL31 K138A-SNAP guided photoablation had no significant effect on Histamine, LY344864 (a serotonin 5-HT1F receptor agonist) or Chloroquine evoked itch (Figure 2f). Finally, to assess the specificity of photoablation to itch sensation, we examined other sensory modalities after treatment. Using the Hot Plate test to assay thermal sensitivity (Figure $2 \mathrm{~g}$ ) and calibrated von Frey filaments to measure mechanical sensitivity (Figure $2 \mathrm{~h}$ ), we observed no difference in response properties of treated mice, indicating that IL31 guided laser ablation is indeed a selective and effective means of disrupting IL31 evoked itch.

\section{IL31K138A-SNAP-IR700 mediated photoablation and Atopic Dermatitis}

We examined the effects of IL31 K138A-SNAP-IR700 ablation on inflammatory itch using the Calcipotriol mouse model of Atopic Dermatitis ${ }^{35}$. To assess the effectiveness of treatment we monitored three indicators of clinical progression: scratching behavior, skin integrity and skin histology. We first determined whether pretreatment with IL31 K138A-SNAP-IR700 would abolish the development of the disease, and then investigated whether post-treatment, upon establishment of inflammation, could reverse symptoms.

As previously reported ${ }^{35}$, application of Calcipotriol to skin provoked a severe Atopic Dermatitis-like phenotype that was evident as a progressive increase in the number of spontaneous scratching bouts over time, distinct skin damage, and a thickening of the epidermis and cell infiltration (Supplementary Figure 3a-d). Injection of IL31 K138A-SNAP_IR700 and subsequent near IR illumination of the skin for 3 days prior to Calcipotriol application completely abolished the development of all indicators in this model. Thus, scratching behavior remained at baseline levels (Figure 3a), skin thickness and histological characteristics were not altered (Figure $3 b$ and c), skin appeared healthy and typical features of dermatitis such as redness and scaling were entirely absent (Figure $3 d$ ). To control for a pharmacological antagonistic effect of IL31 K138A-SNAP we performed identical experiments in the absence of near IR illumination and observed the normal development of dermatitis-like symptoms (Figures 3a-d). Similarly, near IR light and IR700 alone were also ineffective at blocking the progression of the condition (Supplementary Figure 3e-h).

For IL31 ${ }^{\mathrm{K} 138 \mathrm{~A}}$-guided photoablation to have therapeutic relevance, it must also be effective in reversing already established skin inflammation. We therefore treated mice with Calcipotriol for 7 days until severe symptoms were evident. IL31 K138A-SNAP-IR700 was then injected subcutaneously and near IR light applied to the skin for three consecutive days. 
Strikingly, we observed a rescue of all disease indicators over the course of 1 week. Thus, scratching behavior returned to baseline levels (Figure 3e), and skin thickness (Figure 3f), morphology (Figure $3 \mathrm{~g}$ ) and structure (Figure 3h) became indistinguishable from healthy mice. Such reversal of dermatitis-like symptoms was not evident in control experiments where IL31 K138A-SNAP was applied without subsequent near IR illumination (Figure $3 e-h$ ).

To further improve the clinical applicability of IL $31^{\mathrm{K} 138 \mathrm{~A}}$ guided photoablation, we sought to develop a formulation that would allow for topical, pain-free application of IL31 K138A-SNAP IR700. We selected a water-in-oil micro-emulsion preparation based upon previous evidence that this type of formulation can deliver high molecular weight proteins across the dermal barrier ${ }^{36}$. IL31 ${ }^{\mathrm{K} 138 \mathrm{~A}-\mathrm{SNAP}}$-IR700 was loaded into the aqueous phase of the microemulsion, applied topically, and 20 minutes later skin was illuminated with near IR light. Similar to subcutaneous delivery, topical application of IL31 K138A-SNAP-IR700 both prevented (Figure $3 i-I$ ) and reversed (Figure 3m-p) Calcipotriol provoked dermatitis-like symptoms. This was evident as a return to baseline levels of scratching behavior (Figures $3 \mathrm{i}$ and $\mathrm{m}$ ) and a normalization of skin structure and histology (Figures $3 \mathrm{j}-\mathrm{I}$ and $\mathrm{n}-\mathrm{p}$ ). Thus, molecule guided delivery of a photosensitizer complex allows for on-demand, pain-free control of inflammatory itch.

Generation of a mouse model of the genetic skin disorder Familial Primary Localized Cutaneous Amyloidosis (FPLCA)

FPLCA is a rare, hereditary skin disorder driven by mutations in the IL31 receptors IL31RA and OSMR, and characterized by severe pruritus ${ }^{37}$. We sought to generate a mouse model of FPLCA to further test the therapeutic potential of IL31 K138A-SNAP photoablation in a more chronic and challenging clinical setting. We used CRISPR/Cas9 technology to knock-in an S476F mutation into the II31ra locus, orthologous to the human FPLCA mutation IL31RA S521F 26. From 14 offspring, we obtained one IL31RA ${ }^{-/-}$mouse and one IL31RAS476F mouse that were confirmed by sequencing (Supplementary Figure 4b). Upon expansion of the IL31RAS476F line we observed that both heterozygote and homozygous mice spontaneously developed phenotypes that resembled symptoms of FPLCA. These included a significant increase in spontaneous scratching behavior (Supplementary Figure 5a), deposition of Congo red positive amyloid in the skin (Supplementary Figure $5 \mathrm{c}$ and $\mathrm{d}$ ), thickening of the epidermis, and cellular infiltration of the dermis (Supplementary Figures $5 e-h$ ). Of note, we also observed that IL31 SNAP (Supplementary Figure 5b) and IL31 K138A- 
SNAP (Figure 4a) robustly labelled keratinocytes from IL31RAS476F mice at similar concentrations to those used on wildtype cells.

\section{IL31K138A-SNAP-IR700 mediated photoablation reverses symptoms of FPLCA}

To assess whether targeted photoablation can reverse FPLCA symptoms we applied IL31 K138A-SNAP_IR700 micro-emulsion topically to back skin of IL31RA S476F mice. Strikingly, we observed a significant decrease in spontaneous scratching behavior upon illumination that became evident at 3 days after ablation and continued for the 90 day observation period (Figure 4b). We further characterized histological parameters of FPLCA at 14 days post treatment. All pathological indicators were reversed by photoablation including density of amyloid deposition (Figure 4c-e), infiltration of dermal eosinophilic material (Figure 4f and $\mathrm{g}$ ), and mast cell counts (Figure 4h-j). Moreover, redness, scaling and superficial scaring of skin was visibly improved 14 days after treatment and continued for the 90 day observation period (Figure 4k and I). Importantly, in the absence of illumination, we observed no difference in behavioral or histological indicators of FPLCA, suggesting that the cream on its own does not induce any effect.

\section{IL31K138A-SNAP_IR700 mediated photoablation in a mouse model of psoriasis}

To investigate further the scope of $\mathrm{IL} 31^{\mathrm{K} 138 \mathrm{~A}}$ mediated photoablation, we tested its effectiveness in an additional skin disorder, psoriasis, which is not believed to have a strong dependence upon IL31 signaling ${ }^{38}$. We used the Aldara mouse model of psoriasis where Aldara cream, containing the Toll-like receptor 7-ligand Imiquimod, is applied to the skin. In this model, neither pretreatment nor posttreatment with IL31 K138A-SNAP-IR700 and illumination altered scratching behavior provoked by Aldara cream (Supplementary Figure 6). Similarly, skin thickness, erythema, inflammation and integrity remained unaltered by photoablation as shown by the Psoriasis Area and Severity Index (PASI) (Supplementary Figure 6). Thus, IL $31^{\mathrm{K} 138 \mathrm{~A}}$ guided photoablation is likely ineffective in treating psoriasis.

\section{Mechanism of action of IL31K138A-SNAP - mediated photoablation}

To investigate the mechanism underlying IL31 K138A-SNAP_IR700 mediated photoablation we focused our attention on the three major cell populations which express IL31 receptor complexes in the skin; cutaneous dendritic cells (specifically Langerhans cells), keratinocytes and free nerve endings innervating the skin 13, 39-41. Again, we performed photoablation by applying IL31 ${ }^{\mathrm{K} 138 \mathrm{~A}-S N A P_{-} I R 700}$ to the back skin and then analyzed samples from illuminated or control non-illuminated skin at 1 day, 1 week or 1 month post treatment. 
We first considered cutaneous dendritic cells. Skin cell suspensions were labelled with antibodies against Langerin and $\mathrm{MHCll}$, and quantified by flow cytometry. We observed no difference in any of the populations of Langerin and $\mathrm{MHCll}$ positive cells (Langerin ${ }^{+} / \mathrm{MHCll}^{-}$ resident inactivated dendritic cells, Langerin $/ \mathrm{MHCll}^{+}$Langerhans cells, Langerin $/ \mathrm{MHCll}^{+}$ dermal migratory dendritic cells) at any time point after ablation (Figures 5a-d, q, and Supplementary Figure $7 \mathrm{a}$ and b). Turning to keratinocytes, we labelled skin sections with an antibody against Keratin 14 and quantified cellular density. Again, we observed no difference in keratinocyte numbers at any point after ablation (Figures $5 e-h, r$, and Supplementary Figure 7c and d). In agreement with these measures we also detected no difference in the number of TUNEL positive cells at 1 day, 1 week and 1 month post treatment, indicating that illumination does not provoke additional apoptosis in skin (Supplementary Figure 7e-j).

We next examined innervation of the epidermis using the pan-neuronal antibody Pgp9.5 to label nerve endings in skin sections. At one day after treatment we observed no difference in the number of free nerve endings crossing the dermal epidermal junction (Figure $5 \mathrm{i}, \mathrm{j}$ and s). However, at one week and one month post ablation, a marked reduction in epidermal innervation density was evident (Figure 5k-n and s). Similarly, photoablation also provoked a prominent reduction in numbers of epidermal nerve fibers in skin taken from Calcipotriol treated mice (Figure 50-p and s). Importantly, the density of presumed non-pruriceptive fibers innervating hair follicles and dermal structures was not reduced by IL31 K138A-SNAP IR700 treatment, supporting behavioral observations that the approach is specific to itch. Finally, to determine whether nerve ablation alone (without influencing other cells in the skin) is sufficient to reduce scratching behavior, we performed photoablation directly on the proximal sciatic nerve. IL31 ${ }^{\mathrm{K} 138 \mathrm{~A}-\mathrm{SNAP}-I R 700}$ was applied to the sciatic nerve at mid-thigh level and scratching/biting behavior evoked by IL31 injection of the innervation territory in the calf was monitored. Upon illumination of the sciatic nerve we observed a significant reduction in IL31 evoked behavior that was not evident in non-illuminated control animals. Thus, IL31 K138A-SNAP guided photoablation triggers retraction of pruriceptive sensory neurons in the skin, thereby alleviating scratching and the chain of events that leads to inflammation.

\section{Discussion}

Chronic itch is a common and often overlooked condition that is difficult to treat and exerts a substantial societal burden ${ }^{42,43}$. Here we describe a novel approach to control itch that is highly effective in mouse models of Atopic Dermatitis and the genetic skin disease FPLCA. 
Exploiting the natural selectivity of the IL31 ligand to its receptors on itch sensing cells in the skin, we design an IL31 derivative that enables delivery of a photosensitizer to itch sensors in vivo without causing itch in itself. Through illumination of treated skin, we are able to reverse behavioral and histological indicators of atopic dermatitis and FPLCA from a single treatment regime. We demonstrate that this is dependent upon photosensitizer induced retraction of itch sensing neurons from the epidermis, and thus silencing of the neuronal driver of scratching at its source in the skin.

Current therapeutic options for alleviating chronic itch rely on treatments that aim at controlling skin barrier function and inflammation rather than targeting itch itself ${ }^{44}$. For example, in atopic dermatitis patients, initial treatment involves the use of topical moisturizers, emollients and corticosteroids, followed by systemic treatments such as oral immunosuppressants or UV therapy if disease worsens ${ }^{4,43}$. All of these approaches suffer from severe adverse effects, and a significant problem in treating chronic itch is continued adherence to therapy, with most patients abandoning treatments within a year ${ }^{44}$. Recently, the first mechanism based targeted therapy for atopic dermatitis was approved in the US, a human monoclonal antibody against the interleukin-4 receptor alpha subunit named Dupixent ${ }^{45-47}$. In addition, further clinical trials are also underway for a humanized antibody against IL31RA (Nemolizumab) ${ }^{30,31}$, highlighting the potential of biologics in treating itch. It is currently not known what the long term effectiveness and safety of these treatments will be, and the cost of repeated treatment is likely to restrict their use to only the more severe cases of atopic dermatitis ${ }^{45,48}$.

The photoablation approach we describe here has several conceptual differences to the aforementioned strategies for treating pruritic diseases. Firstly, photoablation targets cells rather than single molecules, thus bypassing the enormous molecular complexity that underlies itch. A major difficulty in selecting targets for new antipruritic medications is the molecular redundancy that is inherent to itch sensation. By inactivating the integrating unit of itch, the sensory neuron, rather than individual molecules in the itch pathway, we circumvent this redundancy. Secondly, our data indicates that a single treatment regime with photosensitizer and light allows for long-lasting alleviation of scratching, thus facilitating skin healing. Indeed we observed reversal of IL31-evoked itch that persisted throughout an 8 week observation period, and in the FPLCA model, behavioral and histological indicators were normalized for at least 3 months. In the Calcipotriol model of dermatitis we also observed recovery that continued for the maximal ethically acceptable observation period. Thirdly, through local, on-demand application of ligand-photosensitizer complex and light, 
we are able to achieve precise control of drug action, thus avoiding systemic side effects and allowing therapy to be tuned to disease severity. Further development could also allow for systemic delivery of the photosensitizer complex combined with local application of light. Finally, we have developed an engineered ligand, rather than an antibody or small molecule to target cells. We chose this approach because we wished to exploit the naturally high affinity of IL31 to its receptor, and because of ease of production of recombinant ligand. A similar approach could therefore by readily adapted to other cell surface receptors such as for example IL17 receptors in psoriasis, without the need for extensive screening to identify novel binders. Moreover, existing antibodies or small molecules that target membrane receptors could also be co-opted to deliver photosensitizers, analogous to delivery methods developed for photodynamic therapy of cancer ${ }^{49}$.

Beyond atopic dermatitis, IL31 has been implicated in a number of other pruritic diseases such as prurigo nodularis, cutaneous T-cell lymphoma, and chronic idiopathic pruritus ${ }^{15,17}$, 18, 50, and targeted photoablation could conceivably also have therapeutic efficacy in these disorders. To further examine the scope of IL31 mediated photoablation, we chose to focus on the rare genetic skin disease FPLCA, which is driven by mutations in the IL31 receptor complex ${ }^{24-27}$. FPLCA is characterized by severe pruritus and localized deposition of amyloid in the dermal papilla ${ }^{23}$, both of which are highly distressing and impact profoundly on the quality of life of patients. There are no effective treatment options for FPLCA and management of the disorder relies upon topical application of corticosteroids, UV phototherapy and systemic treatment with immunosuppressants ${ }^{23}$. Here we describe the first mouse model of FPLCA, and demonstrate that mutant IL31RA ${ }^{\text {S476F }}$ mice develop phenotypes that are remarkably similar to human symptoms. Thus we observed a significant increase in spontaneous scratching behavior, damage and inflammation of the skin, and deposition of amyloid. We did not explore in detail the molecular mechanisms that underlie FPLCA, instead focusing on gauging the therapeutic potential of IL $31^{\mathrm{K} 138 \mathrm{~A}}$ mediated photoablation. Indeed we found that a single treatment regime led to a substantial improvement in disease progression that persisted throughout the 3 month observation period. We now expect that the IL31RA ${ }^{\mathrm{S} 476 \mathrm{~F}}$ mouse model will allow for further mechanistic investigations, and will also enable preclinical testing of other emerging IL31 orientated medications such as humanized IL31RA antibodies ${ }^{30,31}$ and JAK inhibitors ${ }^{42,51}$ for treating FPLCA.

IL31 receptor complexes are expressed by cutaneous dendritic cells, keratinocytes, and free nerve endings in the skin ${ }^{13,39-41}$, and hypothetically, IL $31^{\mathrm{K} 138 \mathrm{~A}}$ mediated photoablation could 
induce cell toxicity in any of these cell types. Intriguingly, our analysis of these populations post ablation points towards a selective and sustained loss of neuronal innervation by

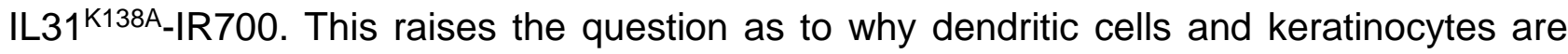
spared by the treatment. One possibility is that there is a transient loss of these cells which we are not able to detect because of ongoing cell turnover and re-infiltration of the skin. However, we did not observe any increase in TUNEL positive cells in the skin after photoablation suggesting that there is not a reduction in these cells through apoptosis. An alternative explanation is that sensory neurons, as excitable cells, are more susceptible to photosensitizer induced damage than dendritic cells or keratinocytes. Indeed, we have previously observed that exposure of sensory neurons to the photosensitizer fluorescein leads to a rapid loss of action potential firing and an increase in resting membrane potential ${ }^{52}$, which could act as an initial trigger to promote neuronal toxicity.

Our data also place sensory neurons as a critical integration point in the transduction of inflammatory itch, and support growing evidence that neurons represent an attractive target for treating pruritic skin diseases ${ }^{1,42,53}$. Of note, only a small proportion of sensory neurons express IL31 receptors ${ }^{54-56}$, and together with previous genetic ablation experiments ${ }^{54}$ our analysis indicates that these neurons are not overtly sensitive to acute application of other pruritogens such as histamine and chloroquine. Intriguingly, recent evidence indicates that these neurons are sensitized by activation of interleukin-4 receptor alpha (the target for the human monoclonal antibody Dupixent), and JAK1 signaling, and that this may promote their involvement in inflammatory itch ${ }^{42}$. Indeed, atopic dermatitis is known to have a prominent neuronal component 5,57, and it has also been argued the FPLCA could represent a neurodermatitis rather than a primary disorder of keratinoctyes ${ }^{25}$. Data presented here suggest that IL31RA expressing sensory neurons are the key drivers of these disorders, and that their removal through photoablation is a powerful means of reversing disease progression.

Long-term elimination of the sensory nerve endings that signal itch could be considered a relatively excessive measure for treating pruritus. However, several other therapies share similar functional principles to IL31 ${ }^{\mathrm{K} 138 \mathrm{~A}}$ mediated photoablation but without the selectivity that ligand targeting offers. These include the use of psoralens as photosensitizers in UV therapy (PUVA) ${ }^{58}$, and the topical application of capsaicin ${ }^{59-61}$, both of which promote the retraction of nerve fibers in the skin ${ }^{62,63}$. These treatments are however associated with adverse effects such as burning pain, increased itchiness and erythema, limiting patient compliance 58,64 . Moreover, non-selective denervation may also lead to a loss of other 
sensations in addition to itch. By targeting directly a subset of pruriceptors using an engineered ligand and near infrared illumination we circumvent these issues, allowing for local on demand treatment of itch. Further development of the technology to target other membrane receptors and neuronal subsets may allow for treatment of a broad range of sensory disorders spanning itch and pain.

\section{Acknowledgments}

We thank Pedro Moreira of EMBL Transgenic Services, Cora Chadick of EMBL Flow Cytometry Facility, Violetta Paribeni and Matteo Gaetani for technical support of our work. We also acknowledge the assistance of the Protein Expression and Purification Core Facility for the generation of IL31 SNAP, IL31 1 K138A-SNAP and Cas9. This work was funded by EMBL and Fondazione Telethon.

\section{Conflict of interest statement}

The authors declare no conflict of interest.

\section{Methods}

\section{Animals}

Wild type, IL31RA ${ }^{-/,}$, and IL31RA ${ }^{S 476 F}$ mice were used at age of 8-10 weeks for all the behavioral studies. 1-3 day-old mice were used for primary keratinocyte culture. All mice were bred and maintained at the EMBL Neurobiology and Epigenetic Unit, Rome, in accordance with Italian legislation (Art. 9, 27. Jan 1992, no 116) under license from the Italian Ministry of Health, and in compliance with the ARRIVE guidelines. IL31RA ${ }^{-/,}$and IL31RA ${ }^{\text {S476F }}$ mice have been deposited in the European Mouse Mutant Archive. 
cDNAs for murine IL31 and SNAP tag were cloned into pETM-11 vector and expressed in E.Coli as fusion protein, carrying a His6-tag at the $\mathrm{N}$-terminus. To generate the mutant IL31 ${ }^{\mathrm{K} 138 \mathrm{~A}-\mathrm{SNAP}}$, site directed mutagenesis was performed according to the manufacturer's instruction (Agilent, \#200555). The proteins were isolated from inclusion bodies, solubilized, refolded, and purified using a Ni-NTA resin (Qiagen, \#30210). Eluted fractions were then pooled, concentrated and stored for further analysis.

\section{Synthesis of BG-IR700}

3mg of IRDye $® 700 D X N$-hydroxysuccinimide ester fluorophore (LI-COR Biosciences $\mathrm{GmbH}$, Bad Homburg, Germany) were dissolved in $150 \mu \mathrm{l}$ DMSO and treated with 1.5mg BG-PEG $11-\mathrm{NH}_{2}$ and $5 \mu \mathrm{l}$ diisopropylethylamine. After $1 \mathrm{~h}$, the product BG-PEG 11- $^{-}$ IRDye®700DX was purified by HPLC using a Waters Sunfire Prep C18 OBD 5 $\mu$ M; $19 \times 150$ mm column using $0.1 \mathrm{M}$ triethylammonium acetate (TEAA)] $(\mathrm{pH} 7.0)$ and $0.1 \mathrm{M}$ TEAA in water/acetonitrile 3:7 $(\mathrm{pH} \mathrm{7.0)}$ as mobile phases $A$ and $B$, respectively. A linear gradient from $100 \%$ A to $100 \%$ B within 30 minutes was used. The fractions containing the product were lyophilized.

\section{Primary Keratinocyte culture}

Primary keratinocytes were isolated from 1-3 day-old wild type, IL31RA ${ }^{-/-}$and IL31RA S476F mice as previously described ${ }^{65}$. Briefly, newborn mouse skin was removed and incubated flat in cold and freshly thawed trypsin overnight, at $4^{\circ} \mathrm{C}$, with the dermis side down. The next day, epidermis was peeled off, triturated and keratinocytes were cultured in serum free media (Invitrogen \#10744-019) on Collagen I (Sigma \# 122-20) - coated dishes (Ibidi \#81151). All experiments were performed on 48-72 hours cultured cells.

\section{In-vitro labeling}

For keratinocyte labelling, $1 \mu \mathrm{M}$ IL31 SNAP or IL31 ${ }^{\text {K138A-SNAP }}$ was coupled with $3 \mu \mathrm{M}$ BG549surf (NEB \# S9112) for 1 hour at 37ㄷ in ClB buffer ( $\mathrm{NaCl} 140 \mathrm{mM}$; KCl $4 \mathrm{mM}$; $\mathrm{CaCl}_{2} 2 \mathrm{mM}$; $\mathrm{MgCl}_{2} 1 \mathrm{mM}$; NaOH 4.55 mM; Glucose 5 mM; HEPES 10 mM; pH 7.4). Cells from wild type 
or IL31RA ${ }^{-/-}$or IL31RA ${ }^{\text {S476F }}$ mice were incubated with the coupling reaction for $15-20$ minutes at $37^{\circ} \mathrm{C}$, then washed 3 times in CIB. $1 \mu \mathrm{g} / \mathrm{ml}$ Hoechst was used for nuclear staining. All images were visualized with a Leica SP5 confocal microscope.

\section{In-vitro photo-ablation}

$1 \mu \mathrm{M}$ IL31 ${ }^{\text {SNAP }}$ and $3 \mu \mathrm{M}$ IR700 were coupled for 1 hour at $37^{\circ} \mathrm{C}$. The coupling reaction was applied for $15-20$ minutes at $37^{\circ} \mathrm{C}$ on primary wild type and IL31RA ${ }^{-/-}$keratinocytes. Cells were then exposed to near infra-red light $(680 \mathrm{~nm})$ at $40 \mathrm{~J} / \mathrm{cm}^{2}$ for 1 minutes. 24 hours after light exposure cell death was assessed by Propidium lodide (PI) staining (Invitrogen \# P3566) and cells were imaged with a Widefield Leica microscope DMI6000B.

\section{In-vivo photo-ablation using injection or micro emulsion as delivery system}

The skin at the nape of the neck of wildtype and IL31RA ${ }^{-/-}$mice was shaved and injected with $5 \mu \mathrm{M}$ IL31 ${ }^{\text {SNAP }}$ or IL31 ${ }^{\mathrm{K} 138 \mathrm{~A}-\mathrm{SNAP}}$ coupled to $15 \mu \mathrm{M}$ IR700 in a $50 \mu$ volume. 20 minutes after the injection, near infra-red light $(680 \mathrm{~nm})$ at $200 \mathrm{~J} / \mathrm{cm}^{2}$ was applied on the injection site for 2 minutes. This procedure was repeated for 3 consecutive days. For von Frey and Hot Plate tests, this procedure was performed on the hind paw using a $20 \mu$ injection volume. All the behavioral experiments were performed 1 week after the last injection.

The micro emulsion was prepared as already described ${ }^{36}$. Briefly, all the components were assembled as follow: Caprylic Triglyceride 81gr; Glyceryl Monocaprylate 27gr; Polysorbate80 12gr; Sorbitan Monooleate $8 \mathrm{gr}$. The micro emulsion was mixed with the coupling reaction (IL31 ${ }^{\mathrm{K} 138 \mathrm{~A}-\mathrm{SNAP}}+\mathrm{IR} 700$ ) at $1: 1$ ratio in $10 \mu$ volume with $5 \mu \mathrm{M}$ as IL31 ${ }^{\mathrm{K} 138 \mathrm{~A}-}$ SNAP final concentration. The mixture was then applied on Calcipotriol-treated mice and FPLCA mice following the same procedure described above for the injection.

\section{Immunofluorescence}

Back skin from 8-10 week-old Black 6/J male mice was dissected and fixed in PFA 4\% overnight at $4^{\circ} \mathrm{C}$. The next day, skin samples were transferred in $30 \%$ sucrose for $3-4$ hours and frozen in OCT. 40- $\mu$ m sections were cut with a cryostat and stained for Rabbit anti- 
Keratin14 (K14) (Covance \# PRB155P) or Rabbit anti-Pgp9.5 (Dako \# Z5116) antibodies (1:200 dilution) in PBS containing 5\% goat serum $+0,3 \%$ Triton-X overnight at $4^{\circ} \mathrm{C}$. Secondary anti-Rabbit Alexa488 antibody was diluted 1:1000 and incubated for 2 hours at $4^{\circ} \mathrm{C}$ in the dark. $1 \mu \mathrm{g} / \mathrm{ml}$ Dapi (Invitrogen \# D1306) was used to stain nuclei. Slides were mounted with Prolong gold antifade (Invitrogen \# P36930) and imaged with a Leica SP5 confocal microscope and analyzed with ImageJ. To quantify $\mathrm{K} 14^{+}$cell, the number of $\mathrm{K} 14^{+}$ per $\mathrm{Dapi}^{+}$cells in the epidermal basal layer was considered and expressed as \%. For Pgp9.5+ fibers quantitation, the region of dermis / epidermis junction was analyzed and only the intra-epidermal free nerve endings per section were considered for counting ${ }^{66}$.

\section{Immunohistochemistry}

Paraffin-embedded back skin from 8-10 week-old Black 6/J or FPLCA male mice was sectioned at $6 \mu \mathrm{m}$ thickness with a microtome. Sections were deparaffinized, hydrated to distilled water and stained with Hematoxylin and Eosin for morphology and cellular infiltration, Toluidine blue for mast cells and Congo Red (Bio-optica \# 04210822) for amyloid deposits. Slides were mounted and imaged with a Microdissector Leica microscope LMD7000.

\section{Flow cytometry}

To analyze differences in Langerhans cell levels upon photoablation, we performed flow cytometry. The whole back skin of wildtype 8-10 week-old Black 6/J male mice was collected at different time points post photoablation (1 day, 1 week, 1 month post) and cutaneous dendritic cells were isolated as previously described ${ }^{67}$. Briefly, the back skin was cut in $1 \mathrm{~cm}$ pieces and digested overnight at $4^{\circ} \mathrm{C}$ in $1 \mathrm{mg} / \mathrm{ml}$ Dispase II (Roche \# 4492078001). The next day, the skin pieces were further digested in an enzymatic solution of $1 \mathrm{mg} / \mathrm{ml}$ Collagenase IV (Worthington \# CLS4 LS004188) and 0,5 mg/ml DNase (Sigma \# DN25) at $37^{\circ} \mathrm{C}$ for 2 hours; finally, the cell suspension was filtered with a $100 \mu \mathrm{m}$ cell strainer, washed with PBS + 0,5 M EDTA + 2\% FBS and centrifuged at 1700 rpm for 5 minutes at $8^{\circ} \mathrm{C}$. Cells 
were re-suspended in $\mathrm{HBSS}+5 \mathrm{mM} \mathrm{MgCl} 2+100 \mu \mathrm{g} / \mathrm{ml}$ DNase $+1 \% \mathrm{BSA}$ and labelled with $0,5 \mu \mathrm{g} / 1 \times 10^{6}$ cells PE anti-mouse CD207 (Langerin) (Biolegend \# 144204) or 0,25 $\mu \mathrm{g} / 1 \times 10^{6}$ cells Alexa Fluor 488 anti mouse IA/IE (MHCII) (Biolegend \# 107616) antibodies, or Dapi (1 $\mu \mathrm{g} / \mathrm{ml}$ ) in PBS $+2 \%$ BSA. Langerhans cells were distinguished by the other cellular populations for their double expression of Langerin and $\mathrm{MHCII}{ }^{67,68}$. The experiments were performed using FACS Aria instrument and data were analyzed using FlowJo software.

\section{SDS-Page and Western blot}

To assess the coupling reaction, 1 and $2 \mu \mathrm{M}$ IL31 ${ }^{\mathrm{K} 138 \mathrm{~A}-\mathrm{SNAP}}$ was coupled with 3 and $6 \mu \mathrm{M}$ BG549 respectively for 1 hour at $37^{\circ} \mathrm{C}$. The coupling reactions were analyzed by SDS-PAGE on a precast acrylamide gel (BioRad \#456-9034), along with the same concentrations of IL31 ${ }^{\mathrm{K} 138 \mathrm{~A}-\mathrm{SNAP}}$ alone. The bands corresponding to the binding of IL31 ${ }^{\mathrm{K} 138 \mathrm{~A}-\mathrm{SNAP}}$ with BG549 were visualized by gel fluorescence. All the samples were visualized by Coomassie staining. For IL31-mediated signaling, back skin from wild-type mice were injected with vehicle (PBS), $5 \mu \mathrm{M}$ IL31 ${ }^{\text {SNAP }}$ or IL31 ${ }^{\mathrm{K} 138 \mathrm{~A}-\mathrm{SNAP}}$. After 1 hour, mice were sacrificed and skin was collected and lysed in Ripa Buffer (Sigma, \#R0278) with proteases inhibitor cocktail (Roche \#11873580001). Protein lysates were quantified by Bradford assay. $30 \mu \mathrm{g}$ total lysate were separated on $10 \%$ SDS-Page gel and transferred to a nitrocellulose membranes (Protran \#10600007). Membrane were incubated with the following antibodies: anti STAT3 (Cell Signaling \#9139), anti phospho STAT3 (Tyr705) (Cell Signaling \#9131), anti MAPK (Cell Signaling \#4695), anti phospho MAPK (Thr202/Tyr204) (Cell Signaling \#9106), anti AKT (Cell Signaling \#4691), anti phospho AKT (Ser473) (Cell Signaling \#9271), anti-Actin (Cell signaling \#4970). Bands were visualized using the ECL detection system (Amersham \#RPN2106); band density was calculated using ImageJ and the levels of phosphorylated proteins were normalized to the total counterpart.

\section{Behavioral testing}

Scratching behavior 
To evaluate the scratching response, 8-10 week-old male wild-type or IL31RA ${ }^{-/}$or

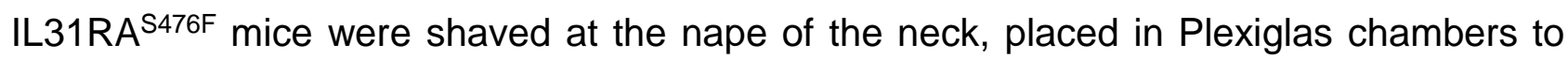
acclimatize (30 minutes), videotaped for 30 minutes and scratching bouts were counted. One bout was defined as an event of scratching lasting from when the animal lifted the hind paw to scratch until it returned it to the floor or started licking it. For IL31-mediated itch, $5 \mu \mathrm{M}$ IL31 ${ }^{\text {SNAP }}$ or IL31 $1^{\text {K138A-SNAP }}$ or native IL31 (Peprotech, \#210-31) were injected intradermal for 1-3 days depending on the experiment. Regarding other itch-mediators, $10 \mathrm{mM}$ histamine (Sigma, \#H7250), 1 mM LY344864 (Abcam, \#ab120592) or 12,5 mM Chloroquine (Sigma, \#C6628) were injected into the back skin of wild type mice previously injected with $5 \mu \mathrm{M}$ IL31 ${ }^{\mathrm{K} 138 \mathrm{~A}-\mathrm{SNAP}}+15 \mu \mathrm{M}$ IR700 with or without near IR illumination. The injection of pruritogens was done 1 week after the last treatment. For long-term reversal IL31 evoked scratching, IL31 was injected 1 day after the last day of illumination and then every week for 8 weeks. $4 \mu \mathrm{g} / \mathrm{ml}$ Calcipotriol-mediated itch was assessed at different time points to monitor dermatitis development, with or without near IR illumination.

von Frey test

Mice were habituated on an elevated platform with a mesh floor for 30 minutes. The plantar side of the hind paw was stimulated with calibrated von-Frey filaments (North coast medical \#NC12775-99) to assess baseline levels of mechanical sensitivity. The hind paw of wildtype mice were then injected for 3 consecutive days with IL31 K138A-SNAP coupled with IR700 with or without near IR light illumination. The stimulation with von-Frey filaments was then repeated 1 week after the last photoablation. The 50\% paw withdrawal thresholds were calculated using the Up-Down method ${ }^{69}$.

Hot Plate test

Mice were injected for 3 consecutive days with IL31 K138A-SNAP coupled with IR700 with or without near IR light illumination. 1 week after the last injection, mice were placed on top of a Hot Plate (Ugo Basile \#35150) that was preset to $52^{\circ} \mathrm{C}$ and the latency to response as 
distinguished by flicking or licking of the hind paw was observed. In order to avoid injury to the mice, a cutoff of 30 seconds was set.

\section{Mouse model for Atopic Dermatitis (AD)}

$4 \mu \mathrm{g} / \mathrm{ml}$ of the vitamin D3 analogue Calcipotriol (Tocris \#2700) was applied topically to the shaved back skin of the mice for 14 or 21 days, depending on the experiment. $0,002 \%$ DMSO was applied as vehicle control for 14 days. Atopic dermatitis development was assessed by scratching behavior over a 30 minute-recording period, skin thickness measurement using a Caliper and histological analysis for cellular infiltration of the treated skin by Hematoxylin \& Eosin staining.

\section{Mouse model for Familiar Primary Localized Cutaneous Amyloidosis (FPLCA)}

The IL31RA ${ }^{S 476 F}$ mouse was generated using cloning-free CRISPR/Cas9 technology as previously described ${ }^{70}$. Briefly, the knock-in point mutation in II31ra locus, responsible for the amino acid substitution S476F 26 , was obtained by zygote pronuclear injection of a mixture of $30 \mathrm{ng} / \mu \mathrm{l}$ Cas 9 protein (Produced and purified by EMBL Protein Expression and Purification Core Facility), 0,61 pmol/ $\mu$ I IL31RA-crRNA (IDT technology), 0,61 pmol/ $\mu \mathrm{l}$ tracrRNA (IDT technology) and $10 \mathrm{ng} / \mu \mathrm{l}$ single strand oligo DNA (ssODN) donor carrying the desired point mutation (C>T) (IDT technology). A 120 nucleotides long ssODN was designed containing two homology arms of 60 nucleotides each, flanking the mutation site. Additional silent point mutations were included in the donor sequence to avoid Cas9mediated cut. Sequences are listed in supplementary figure 4.

\section{Mouse model for psoriasis}

$62,5 \mathrm{mg}$ Aldara 5\% corresponding to $3,125 \mathrm{mg}$ of active component was applied topically to the back skin for 7-10 days as previously described ${ }^{71}$. The development of the disease was monitored and graded using a modified human scoring system Psoriasis Area Severity Index (PASI) which considers skin thickness, erythema and scaling. Scratching behavior was also 
recorded. After 7-10 days, mice were sacrificed and skin was collected for histological analysis.

\section{Nerve photoablation}

The sciatic nerve innervating the hind paw/calf region of $8-10$ week-old male mice was treated with $30 \mathrm{ng} / \mu \mathrm{l}$ TPA to permeabilize the epineural sheath for 15 minutes; then $5 \mu \mathrm{M}$ IL31 ${ }^{\mathrm{K} 138 \mathrm{~A}-\mathrm{SNAP}}+15 \mu \mathrm{M}$ IR700 was applied on the exposed nerve and after 10-15 minutes, near IR light was applied for 45 seconds, except in the control group. Starting from 3 days post treatment, mice were injected with $5 \mu \mathrm{M}$ IL31 SNAP at different days in the paw/calf level, and monitored for scratching expressed as time spent in biting at the injected area.

\section{Statistical analysis}

All statistical data are presented as Standard error of the mean (SEM) along with the number of samples analyzed (n). Student's $t$-test and/or analysis of variance ANOVA followed by the appropriate postHoc test were used. Statistical significance was assumed at $p<0.05$.

\section{References}

1. Ikoma, A., Steinhoff, M., Stander, S., Yosipovitch, G. \& Schmelz, M. The neurobiology of itch. Nat Rev Neurosci 7, 535-547 (2006).

2. Davidson, S. \& Giesler, G.J. The multiple pathways for itch and their interactions with pain. Trends Neurosci 33, 550-558 (2010).

3. Wahlgren, C.F. Itch and atopic dermatitis: an overview. J Dermatol 26, 770-779 (1999).

4. Elmariah, S.B. \& Lerner, E.A. Topical therapies for pruritus. Semin Cutan Med Surg 30, 118-126 (2011).

5. Bautista, D.M., Wilson, S.R. \& Hoon, M.A. Why we scratch an itch: the molecules, cells and circuits of itch. Nat Neurosci 17, 175-182 (2014).

6. Rossbach, K. et al. Histamine $\mathrm{H} 1, \mathrm{H} 3$ and $\mathrm{H} 4$ receptors are involved in pruritus. Neuroscience 190, 89-102 (2011).

7. Liu, Q. et al. Sensory neuron-specific GPCR Mrgprs are itch receptors mediating chloroquine-induced pruritus. Cell 139, 1353-1365 (2009).

8. Reich, A. \& Szepietowski, J.C. Mediators of pruritus in psoriasis. Mediators Inflamm 2007, 64727 (2007). 
9. Takano, N., Arai, I., Hashimoto, Y. \& Kurachi, M. Evaluation of antipruritic effects of several agents on scratching behavior by NC/Nga mice. Eur J Pharmacol 495, 159-165 (2004).

10. Steinhoff, M. et al. Neurophysiological, neuroimmunological, and neuroendocrine basis of pruritus. J Invest Dermatol 126, 1705-1718 (2006).

11. Dillon, S.R. et al. Interleukin 31, a cytokine produced by activated T cells, induces dermatitis in mice. Nat Immunol 5, $752-760$ (2004).

12. Grimstad, O. et al. Anti-interleukin-31-antibodies ameliorate scratching behaviour in NC/Nga mice: a model of atopic dermatitis. Exp Dermatol 18, 35-43 (2009).

13. Bando, T., Morikawa, Y., Komori, T. \& Senba, E. Complete overlap of interleukin-31 receptor $A$ and oncostatin $M$ receptor beta in the adult dorsal root ganglia with distinct developmental expression patterns. Neuroscience 142, 1263-1271 (2006).

14. Diveu, C. et al. Predominant expression of the long isoform of GP130-like (GPL) receptor is required for interleukin-31 signaling. Eur Cytokine Netw 15, 291-302 (2004).

15. Sonkoly, E. et al. IL-31: a new link between T cells and pruritus in atopic skin inflammation. J Allergy Clin Immunol 117, 411-417 (2006).

16. Miyagaki, T. et al. Increased CCL18 expression in patients with cutaneous T-cell lymphoma: association with disease severity and prognosis. Journal of the European Academy of Dermatology and Venereology : JEADV 27, e60-67 (2013).

17. Raap, U. et al. Increased levels of serum IL-31 in chronic spontaneous urticaria. Exp Dermatol 19, 464-466 (2010).

18. Ohmatsu, H. et al. Serum IL-31 levels are increased in patients with cutaneous T-cell lymphoma. Acta Derm Venereol 92, 282-283 (2012).

19. Takaoka, A. et al. Expression of IL-31 gene transcripts in NC/Nga mice with atopic dermatitis. Eur J Pharmacol 516, 180-181 (2005).

20. Raap, U. et al. Correlation of IL-31 serum levels with severity of atopic dermatitis. The Journal of allergy and clinical immunology 122, 421-423 (2008).

21. Singer, E.M. et al. IL-31 is produced by the malignant T-cell population in cutaneous T-Cell lymphoma and correlates with $\mathrm{CTCL}$ pruritus. The Journal of investigative dermatology 133, 2783-2785 (2013).

22. Schulz, F. et al. A common haplotype of the IL-31 gene influencing gene expression is associated with nonatopic eczema. J Allergy Clin Immunol 120, 1097-1102 (2007). 
23. Tanaka, A. et al. New insight into mechanisms of pruritus from molecular studies on familial primary localized cutaneous amyloidosis. The British journal of dermatology 161, 1217-1224 (2009).

24. Lee, D.D. et al. Genome-wide scan identifies a susceptibility locus for familial primary cutaneous amyloidosis on chromosome 5p13.1-q11.2. The British journal of dermatology 155, 1201-1208 (2006).

25. Arita, K. et al. Oncostatin M receptor-beta mutations underlie familial primary localized cutaneous amyloidosis. American journal of human genetics 82, 73-80 (2008).

26. Lin, M.W. et al. Novel IL31RA gene mutation and ancestral OSMR mutant allele in familial primary cutaneous amyloidosis. Eur J Hum Genet 18, 26-32 (2010).

27. Wang, W.H. et al. A new c.1845A-->T of oncostatin M receptor-beta mutation and slightly enhanced oncostatin $M$ receptor-beta expression in a Chinese family with primary localized cutaneous amyloidosis. European journal of dermatology : EJD 22, 29-33 (2012). 28. Zhang, Q., Putheti, P., Zhou, Q., Liu, Q. \& Gao, W. Structures and biological functions of IL-31 and IL-31 receptors. Cytokine Growth Factor Rev 19, 347-356 (2008).

29. Greaves, M.W. \& Khalifa, N. Itch: more than skin deep. International archives of allergy and immunology 135, 166-172 (2004).

30. Ruzicka, T. et al. Anti-Interleukin-31 Receptor A Antibody for Atopic Dermatitis. $N$ Engl J Med 376, 826-835 (2017).

31. Nemoto, O. et al. The first trial of CIM331, a humanized antihuman interleukin-31 receptor $A$ antibody, in healthy volunteers and patients with atopic dermatitis to evaluate safety, tolerability and pharmacokinetics of a single dose in a randomized, double-blind, placebo-controlled study. The British journal of dermatology 174, 296-304 (2016).

32. Amoury, M. et al. Photoimmunotheranostic agents for triple-negative breast cancer diagnosis and therapy that can be activated on demand. Oncotarget (2016).

33. Le Saux, S. et al. Molecular dissection of human interleukin-31-mediated signal transduction through site-directed mutagenesis. J Biol Chem 285, 3470-3477 (2010).

34. Dreuw, A. et al. Characterization of the signaling capacities of the novel gp130-like cytokine receptor. J Biol Chem 279, 36112-36120 (2004).

35. Li, M. et al. Topical vitamin D3 and low-calcemic analogs induce thymic stromal lymphopoietin in mouse keratinocytes and trigger an atopic dermatitis. Proc Natl Acad Sci U S A 103, 11736-11741 (2006). 
36. Himes, R., Lee, S., McMenigall, K. \& Russell-Jones, G.J. Reduction in inflammation in the footpad of carrageenan treated mice following the topical administration of anti-TNF molecules formulated in a micro-emulsion. J Control Release 145, 210-213 (2010).

37. Tanaka, A. et al. The molecular skin pathology of familial primary localized cutaneous amyloidosis. Exp Dermatol 19, 416-423 (2010).

38. Neis, M.M. et al. Enhanced expression levels of IL-31 correlate with IL-4 and IL-13 in atopic and allergic contact dermatitis. J Allergy Clin Immunol 118, 930-937 (2006).

39. Horejs-Hoeck, J. et al. Dendritic cells activated by IFN-gamma/STAT1 express IL-31 receptor and release proinflammatory mediators upon IL-31 treatment. J Immunol 188, 5319-5326 (2012).

40. Heise, R. et al. IL-31 receptor alpha expression in epidermal keratinocytes is modulated by cell differentiation and interferon gamma. J Invest Dermatol 129, 240-243 (2009).

41. Cornelissen, C., Luscher-Firzlaff, J., Baron, J.M. \& Luscher, B. Signaling by IL-31 and functional consequences. Eur J Cell Biol 91, 552-566 (2012).

42. Oetjen, L.K. et al. Sensory Neurons Co-opt Classical Immune Signaling Pathways to Mediate Chronic Itch. Cell 171, 217-228 e213 (2017).

43. Mollanazar, N.K., Smith, P.K. \& Yosipovitch, G. Mediators of Chronic Pruritus in Atopic Dermatitis: Getting the Itch Out? Clin Rev Allergy Immunol 51, 263-292 (2016).

44. Osinka, K., Dumycz, K., Kwiek, B. \& Feleszko, W. Novel Therapeutic Approaches to Atopic Dermatitis. Arch Immunol Ther Exp (Warsz) (2017).

45. Dolgin, E. First eczema biologic debuts but price could restrict use. Nat Biotechnol 35, 391-392 (2017).

46. Beck, L.A. et al. Dupilumab treatment in adults with moderate-to-severe atopic dermatitis. N Engl J Med 371, 130-139 (2014).

47. Thaci, D. et al. Efficacy and safety of dupilumab in adults with moderate-to-severe atopic dermatitis inadequately controlled by topical treatments: a randomised, placebocontrolled, dose-ranging phase $2 \mathrm{~b}$ trial. Lancet 387, 40-52 (2016).

48. Furue, M., Yamamura, K., Kido-Nakahara, M., Nakahara, T. \& Fukui, Y. Emerging role of interleukin-31 and interleukin-31 receptor in pruritus in atopic dermatitis. Allergy (2017).

49. Mitsunaga, M. et al. Cancer cell-selective in vivo near infrared photoimmunotherapy targeting specific membrane molecules. Nat Med 17, 1685-1691 (2011). 
50. Cedeno-Laurent, F. et al. Improved pruritus correlates with lower levels of IL-31 in CTCL patients under different therapeutic modalities. Clin Immunol 158, 1-7 (2015).

51. Gonzales, A.J. et al. Oclacitinib (APOQUEL $((R)))$ is a novel Janus kinase inhibitor with activity against cytokines involved in allergy. J Vet Pharmacol Ther 37, 317-324 (2014). 52. Yang, G. et al. Genetic targeting of chemical indicators in vivo. Nat Methods 12, 137139 (2015).

53. Han, L. et al. A subpopulation of nociceptors specifically linked to itch. Nat Neurosci 16, 174-182 (2013).

54. Stantcheva, K.K. et al. A subpopulation of itch-sensing neurons marked by Ret and somatostatin expression. EMBO Rep 17, 585-600 (2016).

55. Cevikbas, F. et al. A sensory neuron-expressed IL-31 receptor mediates T helper cell-dependent itch: Involvement of TRPV1 and TRPA1. J Allergy Clin Immunol 133, 448460 (2014).

56. Usoskin, D. et al. Unbiased classification of sensory neuron types by large-scale single-cell RNA sequencing. Nat Neurosci 18, 145-153 (2015).

57. Wilson, S.R. et al. The epithelial cell-derived atopic dermatitis cytokine TSLP activates neurons to induce itch. Cell 155, 285-295 (2013).

58. Rivard, J. \& Lim, H.W. Ultraviolet phototherapy for pruritus. Dermatol Ther 18, 344354 (2005).

59. Tarng, D.C., Cho, Y.L., Liu, H.N. \& Huang, T.P. Hemodialysis-related pruritus: a double-blind, placebo-controlled, crossover study of capsaicin $0.025 \%$ cream. Nephron $\mathbf{7 2}$, 617-622 (1996).

60. Wallengren, J. \& Klinker, M. Successful treatment of notalgia paresthetica with topical capsaicin: vehicle-controlled, double-blind, crossover study. Journal of the American Academy of Dermatology 32, 287-289 (1995).

61. Stander, S., Luger, T. \& Metze, D. Treatment of prurigo nodularis with topical capsaicin. Journal of the American Academy of Dermatology 44, 471-478 (2001).

62. Anand, P. \& Bley, K. Topical capsaicin for pain management: therapeutic potential and mechanisms of action of the new high-concentration capsaicin 8\% patch. Br J Anaesth 107, 490-502 (2011).

63. Wallengren, J. \& Sundler, F. Phototherapy reduces the number of epidermal and CGRP-positive dermal nerve fibres. Acta Derm Venereol 84, 111-115 (2004).

64. Hong, J., Buddenkotte, J., Berger, T.G. \& Steinhoff, M. Management of itch in atopic dermatitis. Semin Cutan Med Surg 30, 71-86 (2011). 
65. Lichti, U., Anders, J. \& Yuspa, S.H. Isolation and short-term culture of primary keratinocytes, hair follicle populations and dermal cells from newborn mice and keratinocytes from adult mice for in vitro analysis and for grafting to immunodeficient mice. Nat Protoc 3, 799-810 (2008).

66. McArthur, J.C., Stocks, E.A., Hauer, P., Cornblath, D.R. \& Griffin, J.W. Epidermal nerve fiber density: normative reference range and diagnostic efficiency. Arch Neurol 55 , 1513-1520 (1998).

67. Helft, J. \& Merad, M. Isolation of cutaneous dendritic cells. Methods Mol Biol 595, 231-233 (2010).

68. Stoitzner, P., Romani, N., McLellan, A.D., Tripp, C.H. \& Ebner, S. Isolation of skin dendritic cells from mouse and man. Methods Mol Biol 595, 235-248 (2010).

69. Bonin, R.P., Bories, C. \& De Koninck, Y. A simplified up-down method (SUDO) for measuring mechanical nociception in rodents using von Frey filaments. Mol Pain 10, 26 (2014).

70. Aida, T. et al. Cloning-free CRISPR/Cas system facilitates functional cassette knockin in mice. Genome Biol 16, 87 (2015).

71. Di, T.T. et al. Astilbin inhibits Th17 cell differentiation and ameliorates imiquimodinduced psoriasis-like skin lesions in BALB/c mice via Jak3/Stat3 signaling pathway. Int Immunopharmacol 32, 32-38 (2016).

\section{Figure Legends}

Figure 1. IL31 SNAP labelling and photoablation. (a) Representative Coomassie (upper panel) and fluorescence (lower panel) gel showing the binding of IL31SNAP with the fluorescent substrate BG549 at a 1:3 molar ratio. First and fourth lanes represent the binding of 1 and $2 \mu \mathrm{M}$ IL31SNAP respectively, with BG549. Second and last lanes represent the protein IL31 SNAP alone, 1 and $2 \mu \mathrm{M}$ respectively. (b) Primary keratinocyte culture from wildtype and (c) IL31RA ${ }^{-/}$mice labelled with IL31 SNAP + BG549 (in red). Nuclei were stained with Dapi (in blue). Scale bar $20 \mu \mathrm{m}$. (d) Scratching evoked by intradermal injection of SNAP, IL31 or IL31 SNAP in the nape of the neck of wildtype (WT, $n=4$, black bar) and IL31RA ${ }^{-/}$mice ( $n=4$, white bar). * $p<0.05$ (t-Test). (e, f, g) Propidium lodide staining to assess cell death (in red) 24 hours after photoablation was performed on primary wildtype keratinocytes labelled with IL31SNAP-IR700 (e), with IR700 only (f), or IL31RA ${ }^{-/-}$keratinocytes labelled with IL31SNAP-IR700 (g). Insets represent the corresponding brightfield images. Scale bar 50 
$\mu \mathrm{m}$. (h) Scratching behavior evoked by 3 days of injection (arrows) with IL31 SNAP ( $\mathrm{n}=4$, black circle) or IL31 SNAP + IR700 ( $n=4$, red circle) with near IR illumination. Baseline refers to spontaneous scratching before the first injection. * $p<0.05$ (Bonferroni PostHoc test). Error bar indicate standard error (SEM).

Figure 2. Functional analysis of IL31 K138A-SNAP. (a) Primary keratinocyte cultures from wild type and (b) IL31RA ${ }^{-/-}$mice labelled with IL31 K138A-SNAP + BG549 (in red). Nuclei were stained with DAPI (in blue). Scale bar $20 \mu \mathrm{m}$. (c) Representative western blots showing the expression level of AKT, phospho AKT, MAPK, phospho-MAPK, STAT3, phospho STAT3 and Actin (loading control) in skin injected with vehicle (PBS, lane 1), IL31 SNAP (lane 2) and IL31 K138A-SNAP (lane 3). (d) Levels of each protein were expressed as the ratio between the phosphorylated form and the total counterpart and then normalized to the vehicle-treated sample. (e) Scratching response evoked by 3 consecutive days of injection of IL31 SNAP ( $n=4$, black circle) and IL31K138A-SNAP $(n=8$, red circle). * $p<0.05$ (Two-Way Anova followed by Bonferroni test). (f) Scratching response evoked by the injection of vehicle (PBS, $n=4)$ and different pruritogens (IL31, Histamine, LY344864, and Chloroquine CQ) after mice were injected for 3 consecutive days with IL31 K138A-SNAP-IR700, with ( $n=5$, red bar) and without $(\mathrm{n}=4$, black bar) near IR illumination. * $\mathrm{p}<0.05$ (t-Test). (g) Hot Plate test after 3 days of injection of IL31 K138A-SNAP-IR700 into the hind paw of the mice, with ( $\mathrm{n}=4$, red bar) and without ( $n=4$, black bar) near IR illumination. Baseline refers to the thermal latency before the first injection was performed. Bar graphs represent the latency expressed in seconds (s) of the paw withdrawal in response to heat. (h) von Frey test after 3 days of injection of IL31K138ASNAP_IR700 into the hind paw of the mice, with ( $n=4$, red bar) and without $(n=4$, black bar) near IR illumination. Baseline refers to the mechanical threshold before the first injection was performed. Bar graphs represent the force expressed in grams (g) required to trigger a $50 \%$ response. Error bars indicate SEM. Baseline refers to spontaneous scratching before the first injection. Number of scratching bouts was counted over a 30 minutes recording time.

Figure 3. IL31 ${ }^{\mathrm{K} 138 \mathrm{~A}-\mathrm{SNAP}}$ guided photoablation prevents and reverses atopic dermatitislike symptoms. a-d) Prevention of atopic dermatitis-like symptoms (a) Number of scratch bouts in response to 14 days of Calcipotriol treatment in mice pre-injected with IL31K138ASNAP -IR700, with (red circle, $n=7$ ) or without (black circle, $n=8$ ) near IR illumination. * $p<0.05$ (Two-Way Anova followed by Bonferroni test). (b) Skin thickness was measured at day 14 of Calcipotriol treatment ${ }^{*} \mathrm{p}<0.05$ (t-Test). (c) Representative Hematoxylin and Eosin staining of AD skin sections of mice treated with (Top panel) and without (Lower panel) near 
IR illumination. (d) Representative skin pictures of AD mice with (Top panel) and without (Lower panel) near IR treatment. (e-h) Rescue of atopic dermatitis-like symptoms (e) Scratching bouts in response to 21 days of Calcipotriol treatment of mice treated $(n=6$, red circle), or not ( $n=6$, black circle) with near IR illumination. Arrow indicates the day of the first photoablation. * $\mathrm{p}<0.05$ (Two-Way Anova followed by Bonferroni test). (f) Skin thickness at day 21 of Calcipotriol treatment. ${ }^{*} \mathrm{p}<0.05$ (t-Test). (g) Representative Hematoxylin \& Eosin staining of skin sections of AD mice with (Top panel) and without (Lower panel) near IR illumination. (h) Representative skin pictures of AD mice with (Top panel) and without (Lower panel) near IR treatment (i-I) Prevention of atopic dermatitis-like symptoms using a topical delivery system. (i) Scratching bouts in response to 10 days of Calcipotriol in mice pretreated topically with IL31 K138A-SNAP -IR700, with $(n=4$, red circle) and without $(n=4$, black circle) near IR illumination. * $\mathrm{p}<0.05$ (Two-Way Anova followed by Bonferroni test). (j) Skin thickness after 14 days of Calcipotriol treatment. ${ }^{*} \mathrm{p}<0.05$ (t-Test). (k) Representative Hematoxylin \& Eosin staining of skin sections after 10 days of Calcipotriol treatment. (I) Representative skin pictures. (m-p) Rescue of atopic dermatitis symptoms using topical delivery $(\mathbf{m})$ Scratching behavior evoked by 14 days of Calcipotriol in mice topically treated with IL31K138A-SNAP -IR700, with ( $n=7$, red circle) and without $(n=8$, black circle) near IR illumination. Arrow indicates the first day of photoablation treatment. (n) Skin thickness at day 14 of Calcipotriol treatment. * $\mathrm{p}<0.05$ (t-Test). (o) Representative Hematoxylin \& Eosin staining of skin sections after 14 days of Calcipotriol (p) Representative skin pictures. Error bars indicate SEM, scale bars $200 \mu \mathrm{m}$.

Figure 4. IL31 ${ }^{\mathrm{K} 138 \mathrm{~A}-\mathrm{SNAP}}$ guided photoablation reverses indicators of FPLCA. (a) IL31 ${ }^{\mathrm{K} 138 \mathrm{~A}-\mathrm{SNAP}}+\mathrm{BG549}$ (in red) labels primary keratinocytes from IL31RA $446 \mathrm{~F}$ mice. Nuclei are stained with Dapi (in blue). Scale bar $20 \mu \mathrm{m}$. (b) Spontaneous scratching behavior in IL31RA ${ }^{\text {S476F }}$ mice before any treatment (-1) and following topical application of IL31 K138A-SNAP - IR700, with ( $n=5$, red circle) and without $(n=4$, black circle) near IR illumination. * $p<0.05$ (2-Way Anova followed by Tukey test). (c-d) Representative Congo Red staining for amyloid deposits in skin sections of IL31RA $4476 \mathrm{~F}$ mice $(n=3)$ treated topically with IL31 K138A-SNAP IR700 without (c) or with near IR illumination (d); arrows indicate Congo red positive amyloid deposits. Scale bar $50 \mu \mathrm{m}$. (e) Quantification of Congo red staining expressed as percentage of the total number of epidermal cells. ${ }^{*} \mathrm{p}<0.001$ (t-Test). (f-g) Representative Hematoxylin and Eosin staining of skin sections of IL31RA ${ }^{\text {S476F }}$ mice $(n=3)$ topically treated with IL31 K138ASNAP_IR700 without (f) or with illumination $(\mathbf{g})$, showing reduced dermal infiltration of 
eosinophilic material upon photoablation treatment. Scale bar $200 \mu \mathrm{m}$. (h-i) Representative Toluidine blue staining for mast cells in skin sections of IL31RA S476F mice treated topically with IL31 K138A-SNAP - IR700, without $(\mathbf{h})$ and with (i) near IR illumination. Arrows indicate toluidine blue positive mast cells. Scale bar $50 \mu \mathrm{m}$. (j) Quantification of Toluidine blue staining expressed as number of positive mast cells per section (10 sections, $n=2) ;{ }^{*} p=$ 0.002. (k-I) Representative images of skin of IL31RA ${ }^{\text {S476F }}$ mice 90 days after they have been treated topically with IL31 ${ }^{\mathrm{K} 138 \mathrm{~A}-\mathrm{SNAP}}-\mathrm{IR} 700$ in the absence of illumination $(\mathrm{k})$ or with near IR illumination (I). All error bars indicate standard error (SEM).

\section{Figure 5. Epidermal free nerve endings are the cellular target of IL31 ${ }^{\mathrm{K} 138 \mathrm{~A}-\mathrm{SNAP}}$ guided}

photoablation. (a-d) No loss of epidermal migratory dendritic cells upon IL31 K138A-SNAP mediated photoablation. Representative flow cytometry plots showing the percentage of cutaneous dendritic cells isolated from the back skin of mice at 1 day $(\mathbf{a}, \mathbf{b})$ and 1 week (c, d) after topical application of IL31 K138A-SNAP-IR700, with $(\mathbf{a}, \mathbf{c})$ and without $(\mathbf{b}$, d) near IR illumination. Q2 represents epidermal migratory dendritic cells (Langerhans cells, Langerin+/MHCI+ $)$, Q1 represents Langerin+/MHCll- resident inactivated dendritic cells, Q3 represents Langerin $/ \mathrm{MHCII}^{+}$dermal migratory dendritic cells. Percentage of total events $\left(10^{5}\right.$ cells) are indicated in each box. (e-h) No loss of basal keratinocytes upon IL31 K138ASNAP mediated photoablation. Representative immunofluorescence staining of back skin with a K14 antibody at 1 day $(\mathbf{e}, \mathbf{f})$ and 1 week $(\mathbf{g}, \mathbf{h})$ after topical application of IL31 ${ }^{\mathrm{K} 138 \mathrm{~A}-\mathrm{SNAP} \text { - }}$ IR700, with $(\mathbf{e}, \mathbf{g})$ and without $(\mathbf{f}, \mathbf{h})$ near IR illumination. (i-p) Loss of epidermal free nerve endings upon IL31 ${ }^{\mathrm{K} 138 \mathrm{~A}-\mathrm{SNAP}}$ mediated photoablation. Representative immunofluorescence staining of Pgp9.5 positive nerve fibers innervating back skin at 1 day $(\mathbf{i}, \mathbf{j}), 1$ week $(\mathbf{k}, \mathbf{I}), 1$ month $(\mathbf{m}, \mathbf{n})$, or upon establishment of Atopic Dermatitis $(\mathbf{0}, \mathbf{p})$, after IL31 K138A-SNAP_IR700 application. Dotted line represents the dermal-epidermal junction, white arrows indicate epidermal free nerve endings which are lost upon illumination, magenta arrows show innervations around hair follicles which are spared. (q-s) Quantification of the number of Langerhans cells $(\mathbf{q})$, keratinocytes $(\mathbf{r})$, or epidermal free nerve endings $(\mathbf{s})$ at the indicated time point post IL31 ${ }^{\mathrm{K} 138 \mathrm{~A}-\mathrm{SNAP}}$-IR700 application $\left({ }^{* *} \mathrm{p}<0.001, \mathrm{t}-\mathrm{Test}\right)$. (t) Peri-sciatic nerve application of IL31 K138A-SNAP-IR700 reduces IL31 biting behavior upon near infrared illumination ( $n=5$, red circle) compared with no light treated group ( $n=4$, black circle). Arrow indicates the day of nerve photoablation. * $p<0.05$ (2-Way ANOVA followed byTukey test). Scale bars indicate $50 \mu \mathrm{m}$, error bars indicate SEM. 
bioRxiv preprint doi: https://doi.org/101101/225391: this version posted November 27, 2017. The copyright holder for this preprint (which was not certified by peer review) is the author/funder. All rights reserved. No reuse allowed without permission.

a

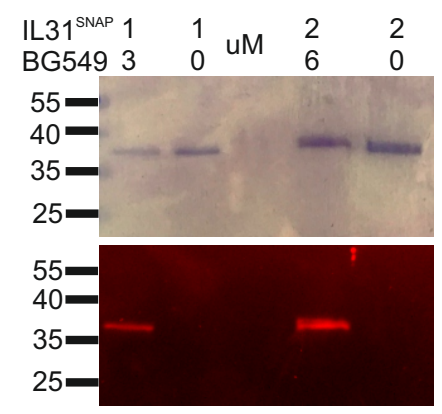

e

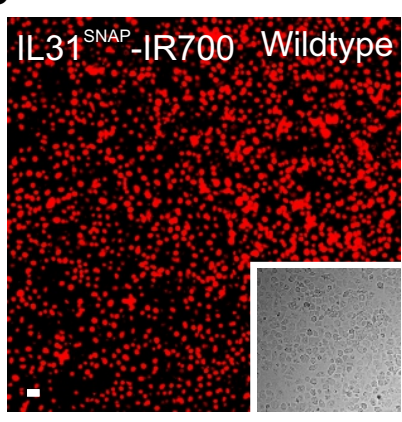

b

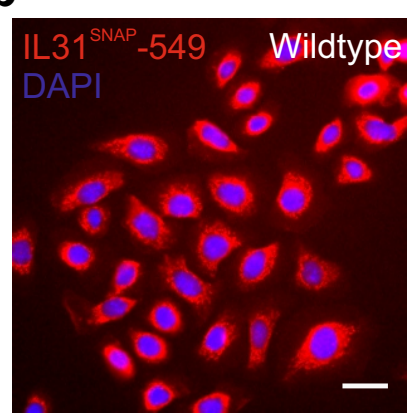

f

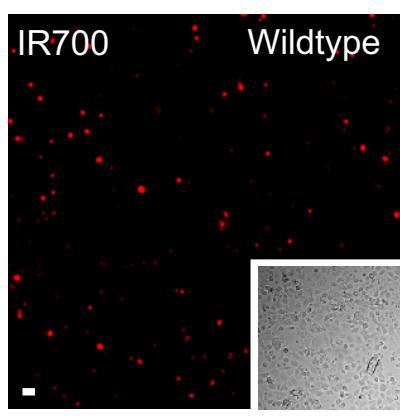

C

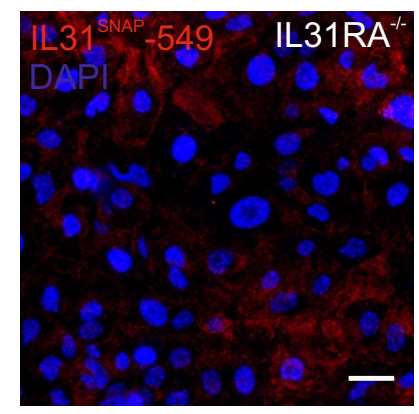

g

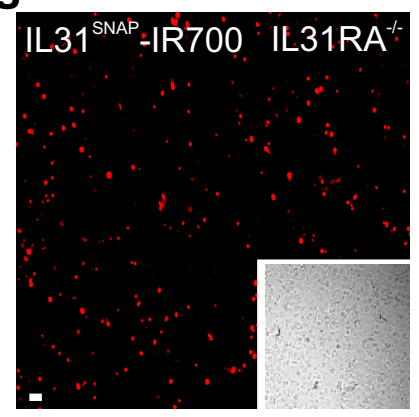

d

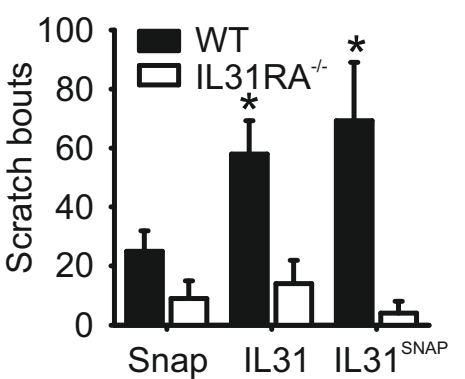

h

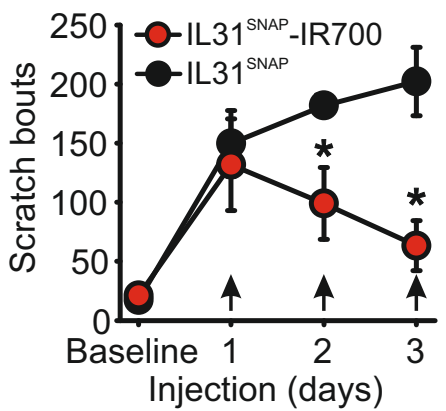


bioRxiv preprint doi: https://doi.org/101101/225391; this version posted November 27, 2017. The copyright holder for this preprint (which was not certified by peer review) is the author/funder. All rights reserved. No reuse allowed without permission.

a

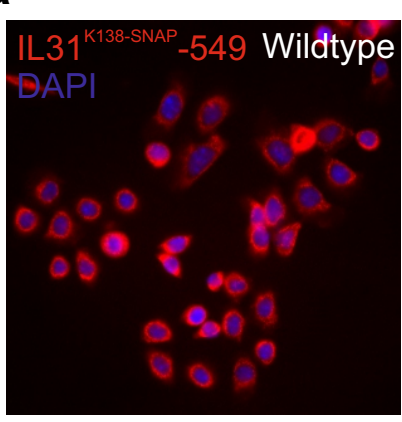

e

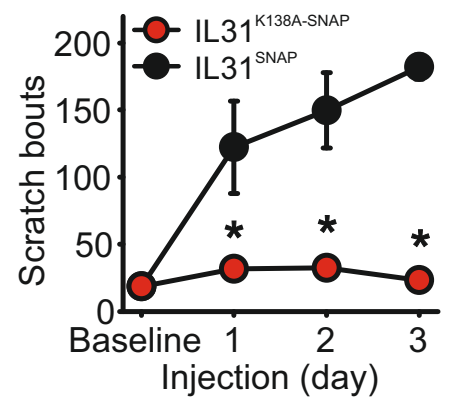

b

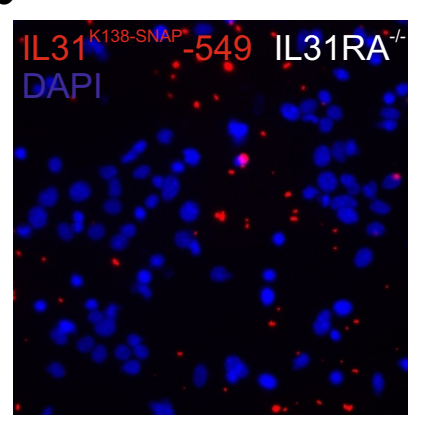

f

Pruritogens

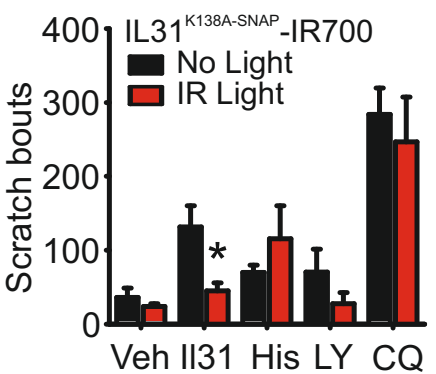

C

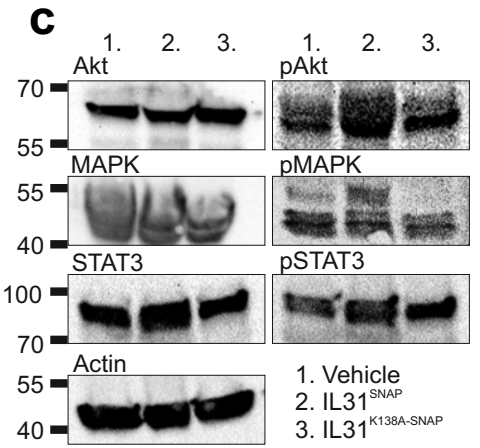

g

Hotplate

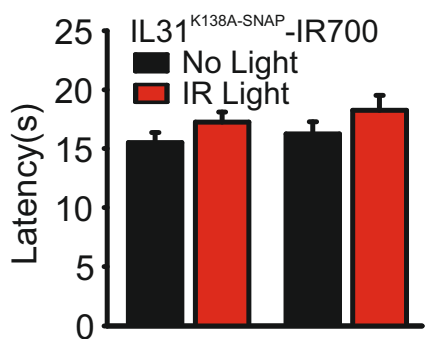

Baseline Post-ablation d

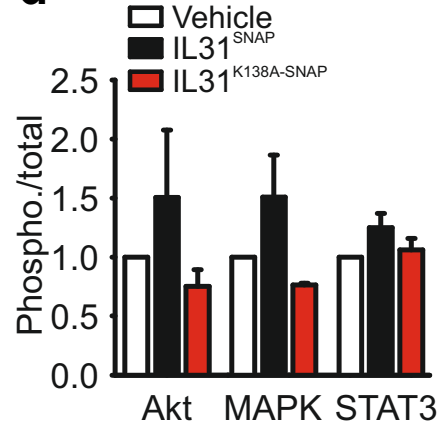

h

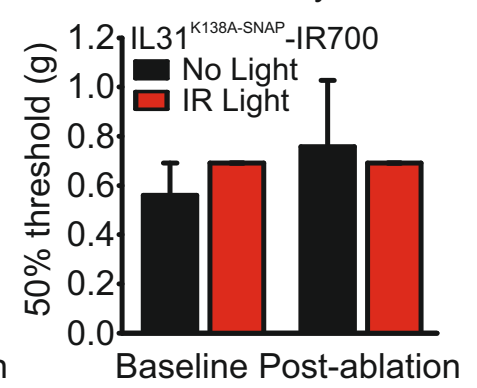


bioRxiv preprint doi: https://doi.org/10.1101/225391; this version posted November 27,2017 . The copyright holder for this preprint (which was not certified by peer review) is the author/funder. All rights reserved. No reuse allowed without permission.

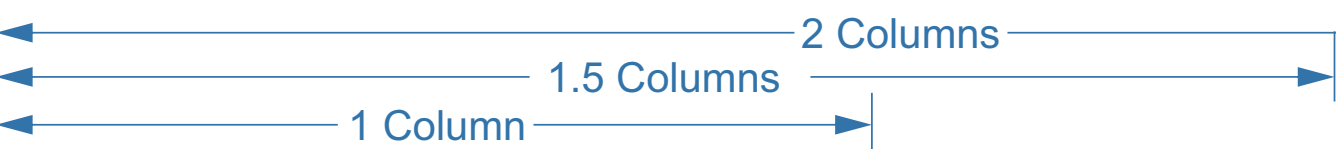

a

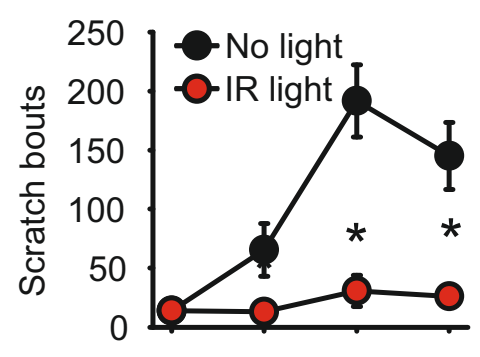

Baseline $1 \quad 7 \quad 14$

Calcipotriol (Days)

e

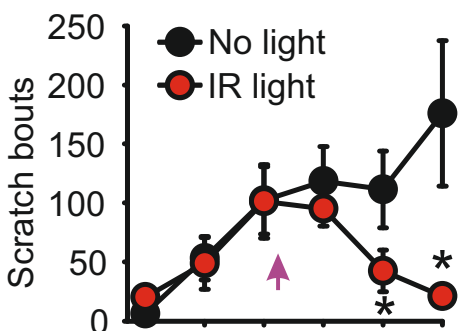

$\begin{array}{lllll}\text { Base } 3 & 5 & 10 & 14 & 21\end{array}$

Calcipotriol (Days)

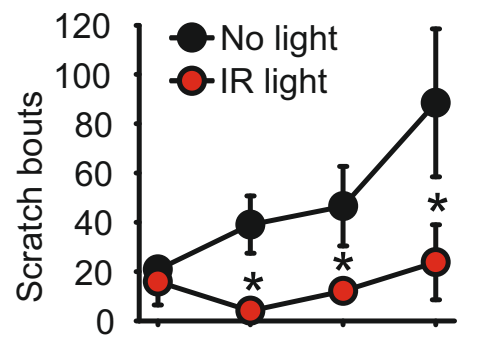

$\begin{array}{lll}\text { Baseline } 1 & 7 & 10\end{array}$

Calcipotriol (Days)

m

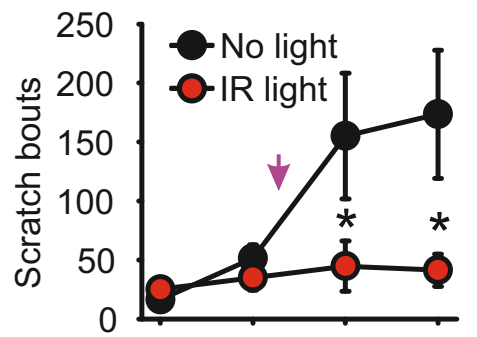

$\begin{array}{lll}\text { Baseline } 3 & 10 & 14\end{array}$ Calcipotriol (Days) b

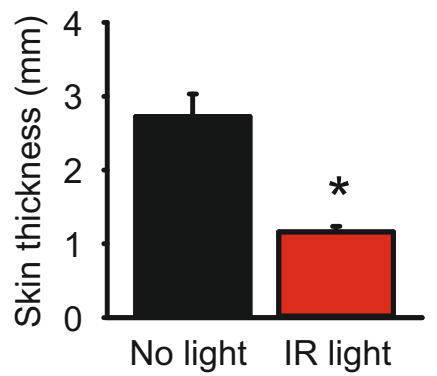

f

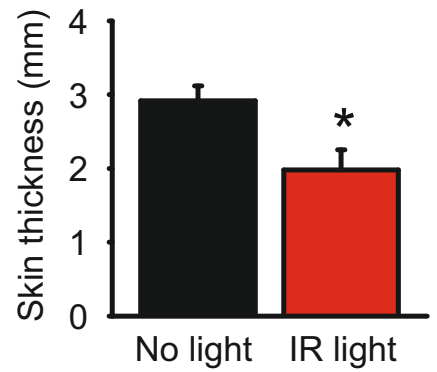

j
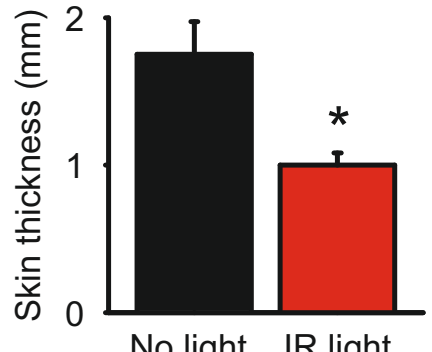

No light IR light

n

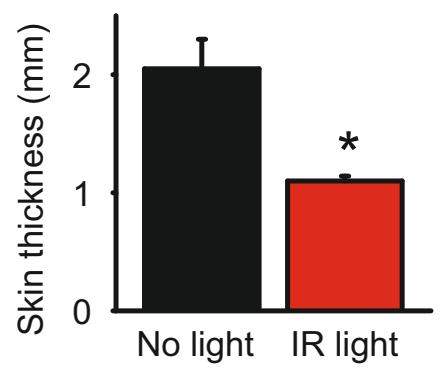

C

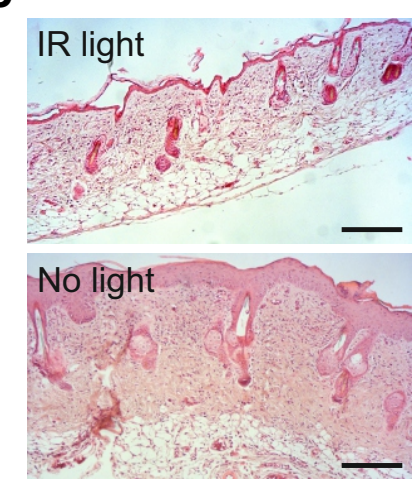

g

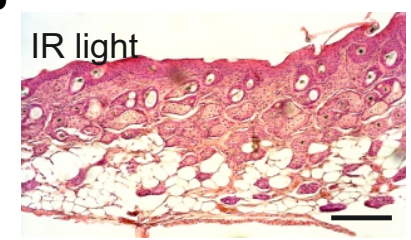

No light

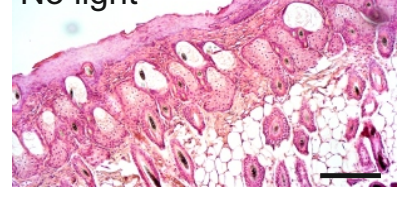

k

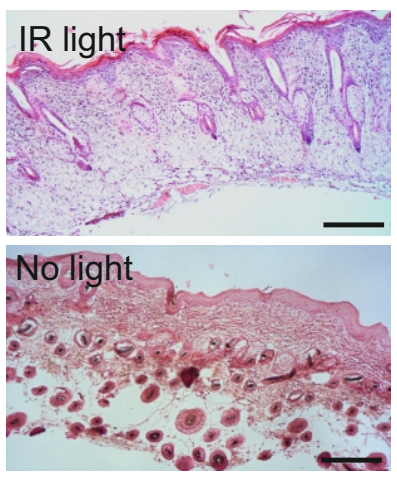

O
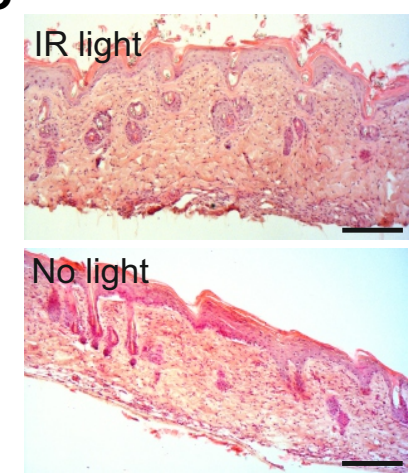

d

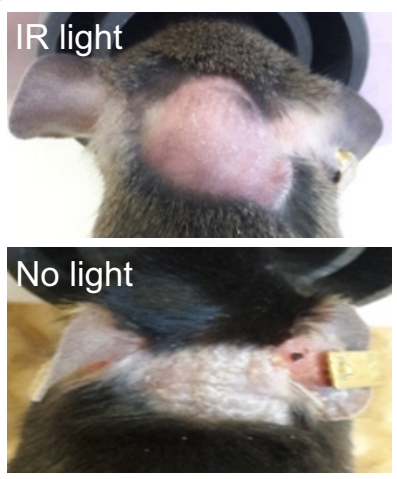

h
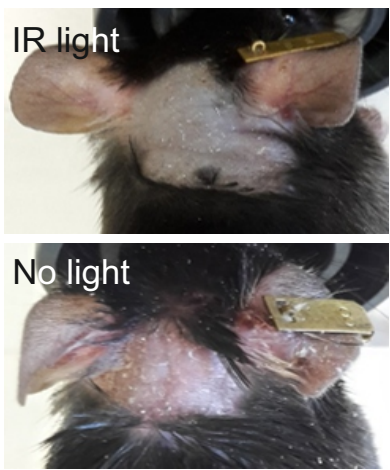

I

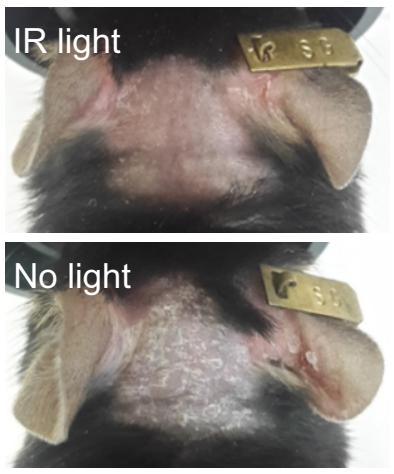

p

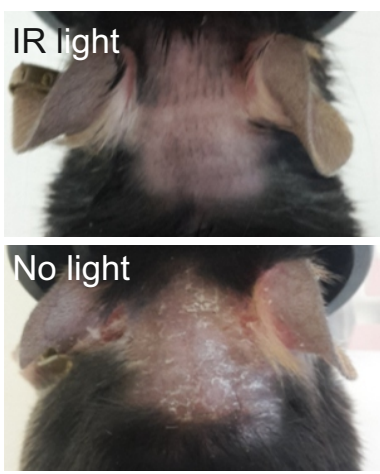


bioRxiv preprint doi: https://doi org/101101/225391; this version posted November 27,2017 . The copyright holder for this preprint (which was not certified by peer review) is the author/funder. All rights reserved. No reuse allowed without permission.

a

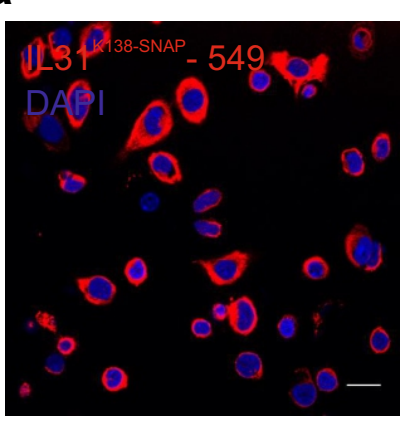

e

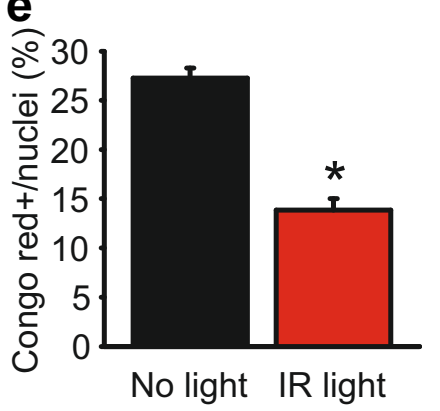

i

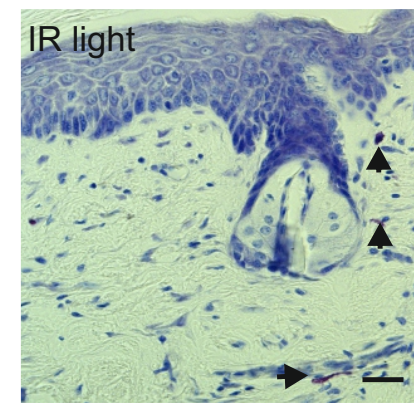

b

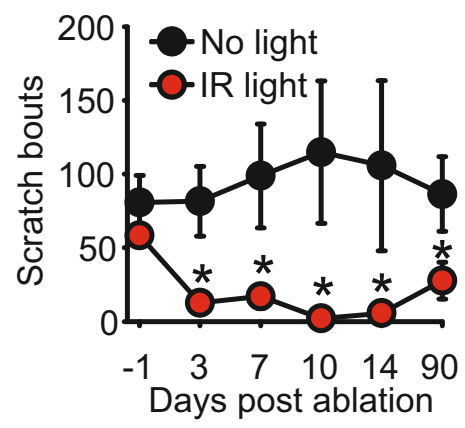

f

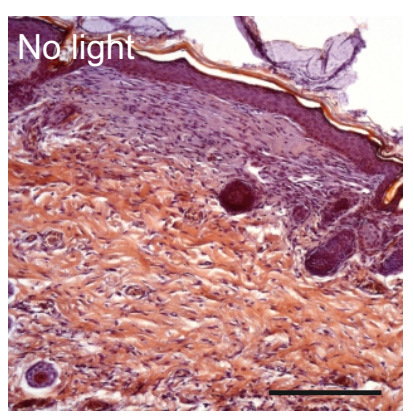

j

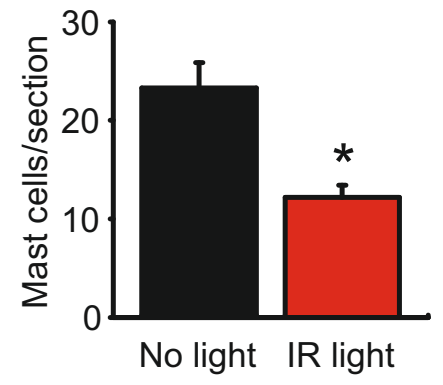

C

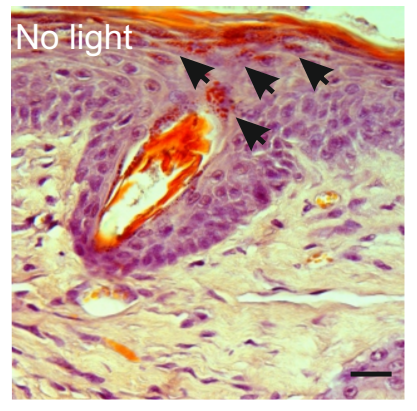

g

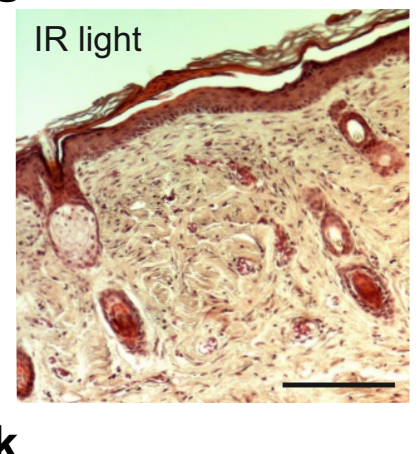

No light

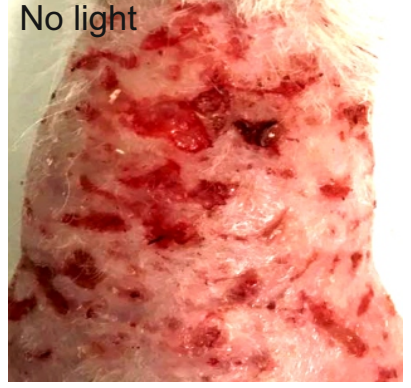

\section{d}

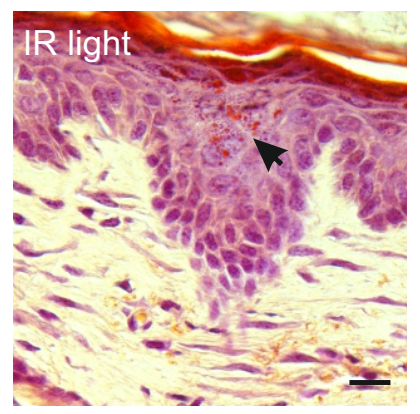

h

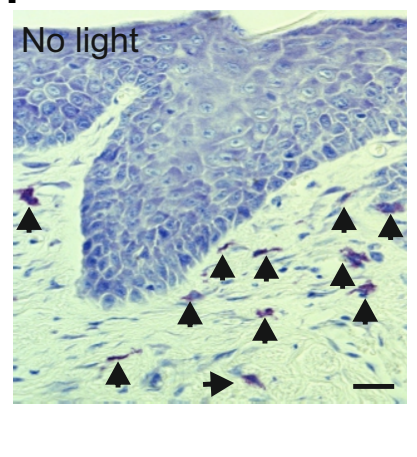

IR light

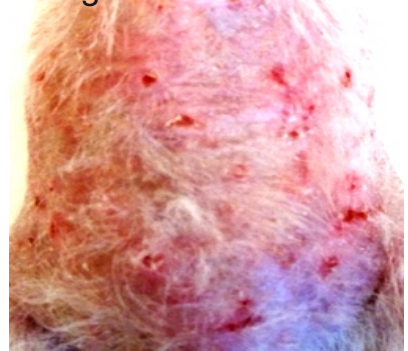


bioRxiv preprint doi: https://doi.org/10.1101/225391; this version posted November 27, 2017. The copyright holder for this preprint (which was not certified by peer review) is the author/funder. All rights reserved. No reuse allowed without permission.

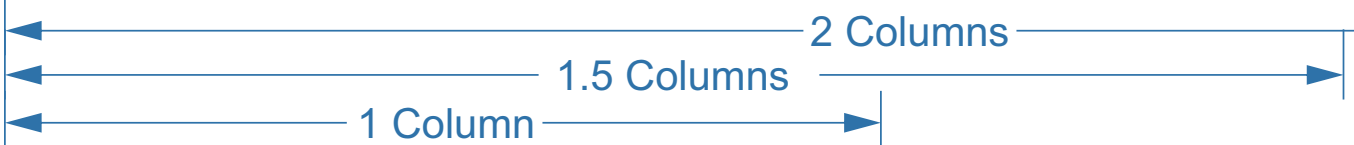

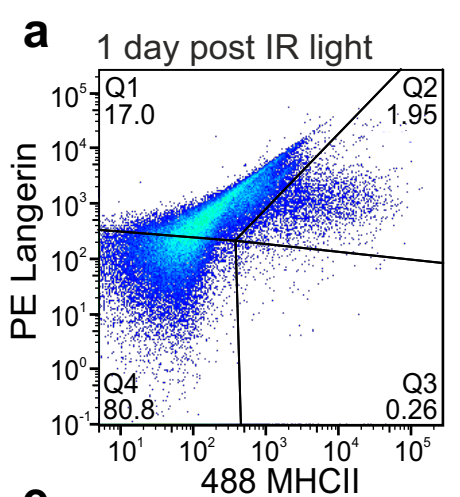

e

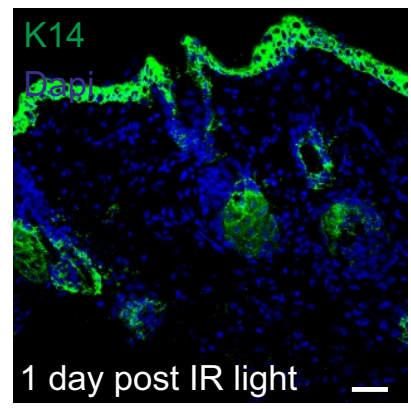

i

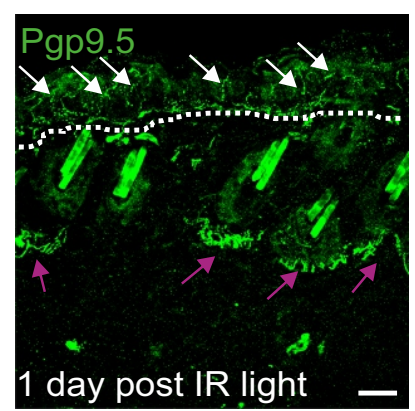

m

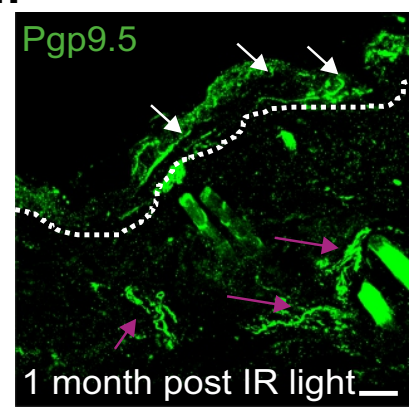

q

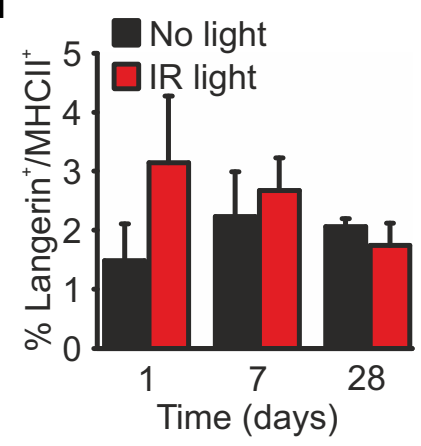

b

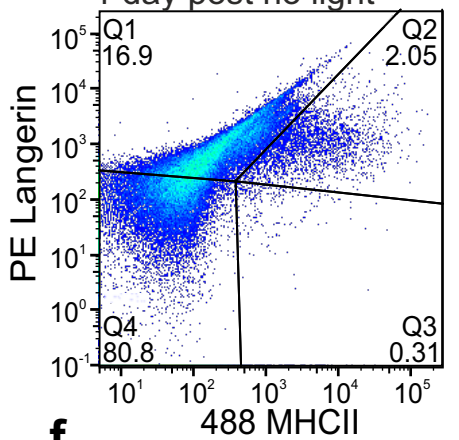

$f$

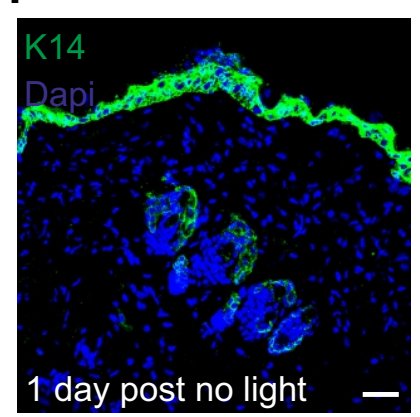

j

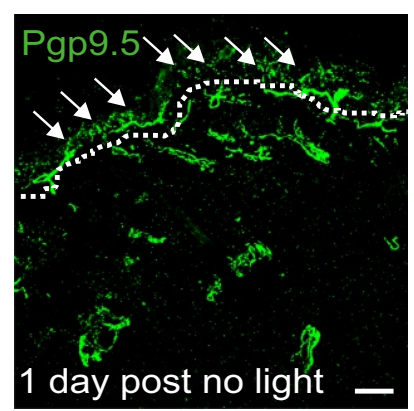

n

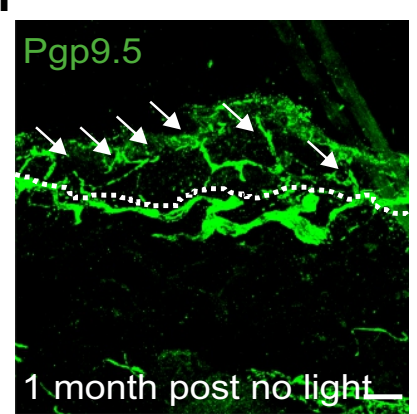

$r$

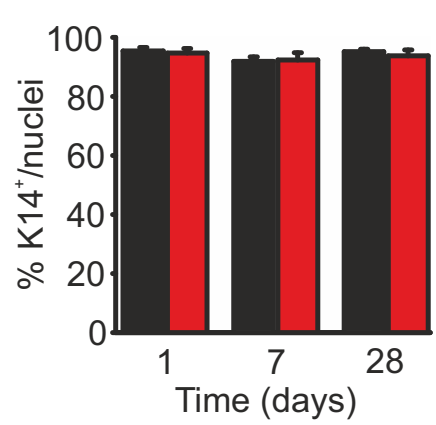

C

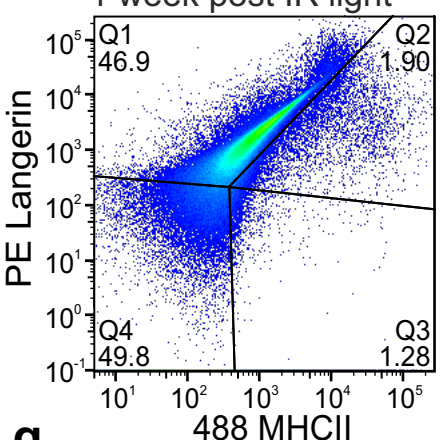

g

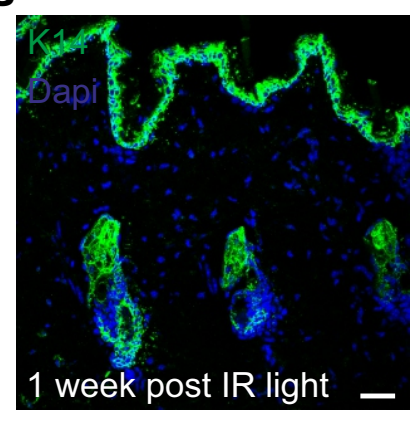

k

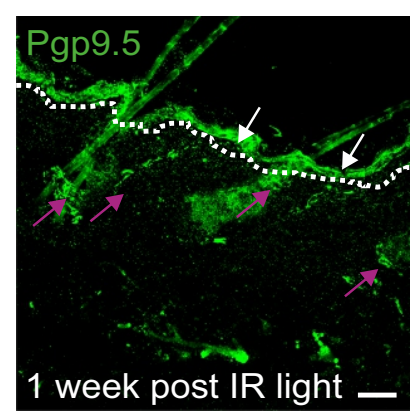

0

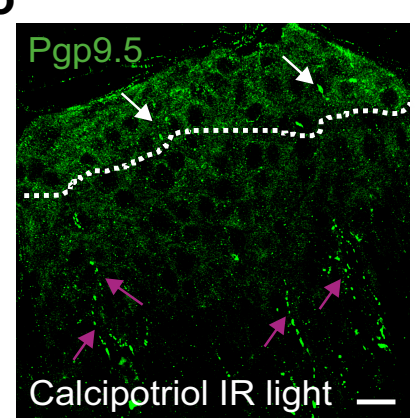

$\mathbf{S}$

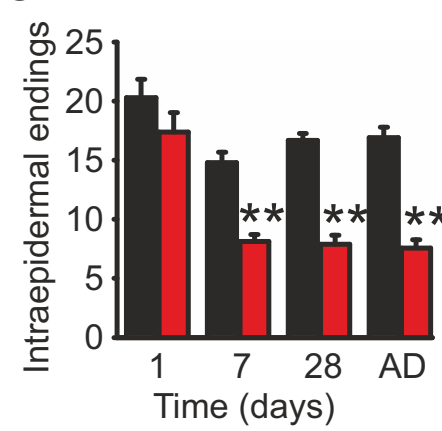

d 1 week post no light

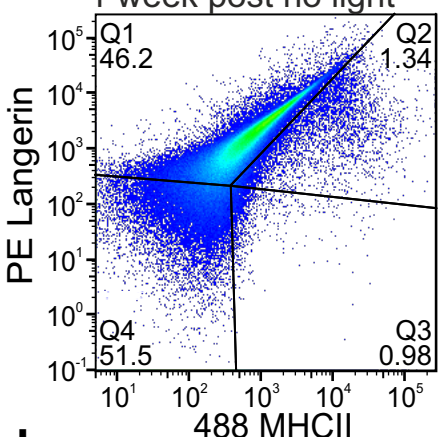

h

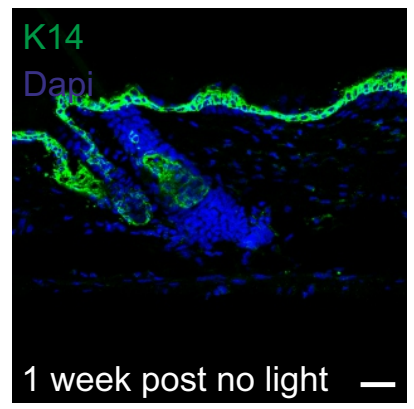

I

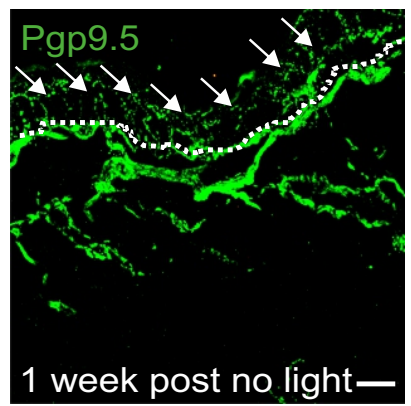

p

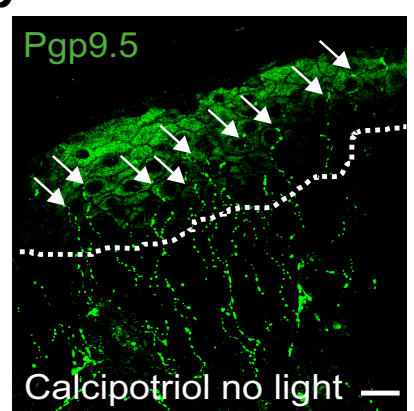

t

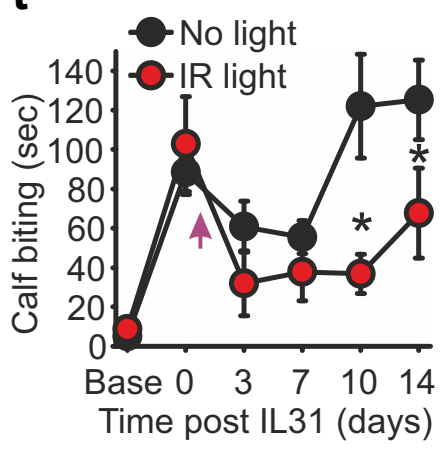

